Supplementary Table 1: Number and percentage of cells in different cellular clusters in diabetic and non-diabetic skin samples

\begin{tabular}{|cccccc}
\hline Cell type & Total number of cells & diabetic & non-diabetic & diabetic \% & non-diabetic \% \\
Endo & 7470 & 1736 & 5734 & 14.79 & 23.16 \\
T cells & 4827 & 1627 & 3200 & 13.87 & 12.93 \\
Fibro A & 4486 & 1610 & 2876 & 13.72 & 11.62 \\
Fibro B & 4002 & 1229 & 2773 & 10.47 & 11.20 \\
SMC & 3494 & 1236 & 2258 & 10.53 & 9.12 \\
NK cells & 3437 & 1208 & 2229 & 10.29 & 9.00 \\
Kera A & 3340 & 1197 & 2143 & 10.20 & 8.66 \\
Myeloid & 2625 & 1004 & 1621 & 8.56 & 6.55 \\
Mast cells & 1000 & 376 & 624 & 3.20 & 2.52 \\
LEC & 682 & 200 & 482 & 1.70 & 1.95 \\
Kera B & 663 & 237 & 426 & 2.02 & 1.72 \\
Melanocytes & 461 & 74 & 387 & 0.63 & 1.56
\end{tabular}




\begin{tabular}{|c|c|c|c|c|c|c|c|c|c|c|c|c|}
\hline Gene name & Endo & T cells & Fibro A & Fibro B & SMC & NK cells & Kera A & Myeloid & Mast cells & LEC & Kera B & Melanocytes \\
\hline PLCG2 & -0.51829 & -1.61827 & -0.89538 & -0.85789 & -0.55076 & -1.51154 & -0.84698 & -0.67542 & -1.222980388 & -0.78138 & & -0.718233163 \\
\hline MT2A & -0.70444 & -1.74069 & -1.34467 & -0.98279 & -1.55382 & -2.68495 & -1.25257 & -1.48945 & -1.496837228 & & & -1.017690984 \\
\hline MT1E & -0.51328 & -1.65455 & -1.20844 & -1.08032 & -1.50535 & -2.65874 & -1.16908 & -0.84116 & & & & -1.22026088 \\
\hline RPS10 & -0.61655 & -0.71939 & -0.65034 & -0.64287 & -0.75537 & -0.7076 & -0.64517 & -0.71343 & & -0.84976 & & \\
\hline TSC22D3 & -0.42221 & -0.69964 & -1.07574 & -0.62338 & & -0.65375 & -0.56204 & -0.76217 & -0.826420517 & & & \\
\hline MT1X & -0.68748 & -1.76328 & -0.83974 & -0.82603 & -1.55297 & -2.943 & -0.74536 & -1.29597 & & & & \\
\hline XIST & -1.56524 & -1.00625 & -1.45624 & -1.02171 & -1.40814 & & -1.69664 & -1.54221 & & -1.4604 & & \\
\hline CFD & & -0.37461 & -0.4973 & -0.6487 & -0.29393 & -0.30952 & -0.76735 & & & & -1.587 & \\
\hline S100A10 & & -0.34742 & -0.63087 & & & -0.28596 & -0.41222 & -0.71745 & -0.627556477 & -0.89269 & & \\
\hline RPS4X & & -0.30438 & & & & -0.30731 & -0.57456 & -0.4485 & & -0.34288 & -0.70079 & \\
\hline HNRNPH1 & -0.2675 & -0.32618 & -0.56399 & -0.31782 & -0.27281 & & & -0.28832 & & & & \\
\hline RPL5 & & -0.30521 & & & & -0.33147 & & -0.26333 & & -0.35603 & -0.29843 & \\
\hline MTRNR2L1 & & & -1.06131 & -1.15493 & -0.86218 & & & -1.02078 & & & & -1.93419504 \\
\hline SELENOW & -0.70607 & & -0.58334 & -0.48046 & & & -0.41271 & & & -0.67499 & & \\
\hline GLUL & & -0.35277 & -0.48511 & -0.43019 & & -0.26844 & & & -0.374979519 & & & \\
\hline EMP1 & -0.63973 & & -0.2882 & & & & & -0.5986 & & -0.95891 & & -0.687732748 \\
\hline MTRNR2L8 & & -1.36495 & -0.93336 & -0.88779 & & -1.1551 & & -0.96418 & & & & \\
\hline PDK4 & -0.9275 & & -0.58305 & -0.52533 & -1.03026 & & -0.29733 & & & & & \\
\hline MT-ND4 & & & -0.34704 & -0.42785 & & & & & -0.369765169 & & -0.9111 & -0.339265244 \\
\hline TXNIP & -0.37089 & -1.15252 & & & & -0.64823 & -0.57049 & -0.33961 & & & & \\
\hline CST3 & & & -0.63124 & -0.31239 & -0.40714 & & -0.57815 & -0.72345 & & & & \\
\hline AMD1 & -0.27481 & & -0.29165 & -0.26657 & -0.31356 & & & & & & & \\
\hline MT-ND4L & & & -0.46317 & -0.47449 & & & & & -0.295184574 & & & -0.434036559 \\
\hline $\mathrm{DCN}$ & & -0.26316 & -0.25791 & & & & & -0.28952 & & & -0.63459 & \\
\hline HSPA8 & & -0.36996 & -0.29255 & & -0.34533 & -0.40919 & & & & & & \\
\hline TPPP3 & & & -0.96786 & -0.6631 & & & -0.80706 & & & -0.61637 & & \\
\hline KLF9 & -0.29088 & & & & -0.39013 & & -0.32722 & -0.4548 & & & & \\
\hline TXN & & -0.26626 & -0.34345 & & -0.27977 & & & & -0.459182072 & & & \\
\hline HSPH1 & & -0.61201 & -0.40378 & & & -0.62171 & & -0.50868 & & & & \\
\hline RPL36A & & -0.27653 & & & -0.27121 & -0.30409 & & -0.33125 & & & & \\
\hline PHLDA1 & -0.32112 & -0.29653 & -0.29581 & & -0.42253 & & & & & & & \\
\hline LGALS1 & & -0.26655 & -0.30962 & & & -0.40176 & & & -0.512993711 & & & \\
\hline PLAC9 & & & -0.90262 & -0.44301 & -0.26511 & & & & & -0.61077 & & \\
\hline FKBP5 & -0.29861 & -0.47995 & & & -0.57148 & -0.62695 & & & & & & \\
\hline CEBPD & -0.4186 & & & & -0.46858 & -0.27758 & & -0.55057 & & & & \\
\hline TMSB4X & & & -0.52524 & & & & & & & -0.36397 & -0.57502 & \\
\hline MINOS1 & & & -0.41778 & -0.41671 & & & -0.27935 & & & & & \\
\hline ISG15 & -0.74242 & & -0.31826 & & -0.67189 & & & & & & & \\
\hline FGL2 & & & -0.67402 & -0.62346 & & & & -0.69652 & & & & \\
\hline KIAA0930 & & & -0.53335 & -0.27507 & & & & & & & & -0.575935045 \\
\hline CRIP2 & & & -0.46312 & & & & -0.33277 & & & -0.42426 & & \\
\hline TAGLN2 & & & -0.5027 & & & & -0.29156 & -0.47576 & & & & \\
\hline MT1A & & & -0.55071 & -0.55483 & -1.29893 & & & & & & & \\
\hline ZFAND5 & & & -0.31068 & & -0.28277 & & & -0.36477 & & & & \\
\hline SAMHD1 & -0.28829 & & -0.39587 & & -0.30211 & & & & & & & \\
\hline HMCN1 & & & -0.40296 & -0.3525 & & & & & & -0.40499 & & \\
\hline FABP5 & -0.53741 & & & & -0.28701 & & & & & & -1.31885 & \\
\hline NOVA1 & & & -0.38276 & -0.40582 & & & & & & & -1.06777 & \\
\hline SRP14 & -0.34471 & & & & & & & & & -0.32999 & -0.29037 & \\
\hline HSP90AA1 & & -0.49823 & & & -0.44947 & & & -0.54222 & & & & \\
\hline DNAJB1 & -0.36478 & & & & -0.72994 & & & -0.54892 & & & & \\
\hline AKAP13 & & & -0.26622 & & & & & -0.32863 & -0.49084144 & & & \\
\hline GSTM3 & & & -0.35572 & -0.28747 & & & -0.4804 & & & & & \\
\hline HES4 & & & -0.38174 & & -0.5264 & & & & -0.64139209 & & & \\
\hline MTRNR2L12 & & & -0.26393 & -0.29888 & & -0.29433 & & & & & & \\
\hline TSC22D1 & & & -0.72355 & & & & -0.2619 & & -0.648557435 & & & \\
\hline MT-CO1 & & & -0.3173 & -0.2892 & & & & & & & -0.6447 & \\
\hline CD55 & & & -0.6514 & -0.85935 & & & & -0.29887 & & & & \\
\hline GSN & -0.3434 & & -0.48334 & & & & -0.32464 & & & & & \\
\hline MT1M & & & -1.29712 & -1.21224 & -1.57625 & & & & & & & \\
\hline HLA-DRB5 & -0.41129 & & & & & -0.40889 & & -0.91061 & & & & \\
\hline DYNLL1 & & -0.29377 & -0.34331 & & & & -0.44079 & & & & & \\
\hline NDUFA11 & & & -0.29797 & -0.2866 & & & -0.25197 & & & & & \\
\hline PLIN2 & -0.70495 & & & & & & -0.4021 & & -0.425369152 & & & \\
\hline DPYSL2 & & & -0.41773 & & & & & & -0.517394542 & & & -0.847981119 \\
\hline PABPC4 & & -0.28949 & & & & & & -0.31016 & -0.427726324 & & & \\
\hline
\end{tabular}


Supplementary Table 2

\begin{tabular}{|c|c|c|c|c|c|c|c|c|c|c|c|c|}
\hline RPL15 & & & & & & & -0.32584 & -0.26255 & & -0.27675 & & \\
\hline BGN & & & -0.48384 & -0.47471 & & & & & & & & \\
\hline C1orf56 & & & & & & -0.27746 & & -0.34128 & & & & \\
\hline ANXA4 & & & -0.40243 & -0.36096 & & & & & & & & \\
\hline NTM & & & & -0.26926 & & & & & -0.787203283 & & & \\
\hline YBX3 & & & & & -0.34187 & & & & -0.490025139 & & & \\
\hline HSPD1 & & & & & -0.2994 & & & -0.53737 & & & & \\
\hline ODC1 & -0.27292 & & & & & & & & & & -1.57202 & \\
\hline GAS1 & & & -0.46334 & -0.37053 & & & & & & & & \\
\hline STMN1 & & -0.27019 & & & & & & & & & -1.79192 & \\
\hline PLP2 & & & -0.27099 & & & & -0.30701 & & & & & \\
\hline MAMDC2 & & & -0.59234 & -0.29675 & & & & & & & & \\
\hline PI16 & & & -0.9364 & -0.86466 & & & & & & & & \\
\hline HSP90AB1 & & & & & -0.25169 & & & -0.2944 & & & & \\
\hline MAFB & & & -0.49643 & -0.35195 & & & & & & & & \\
\hline IFI6 & -0.69019 & & & & -0.66825 & & & & & & & \\
\hline ABLIM1 & & & -0.51869 & -0.4601 & & & & & & & & \\
\hline SPON2 & & & & & & -0.48921 & -0.31641 & & & & & \\
\hline DKK2 & & & -0.38534 & -0.35941 & & & & & & & & \\
\hline VEGFB & & & -0.30213 & -0.37112 & & & & & & & & \\
\hline IFNGR1 & & & & & & & & -0.39753 & -0.469798175 & & & \\
\hline SPARC & & & -0.45873 & -0.74692 & & & & & & & & \\
\hline ESYT2 & & & -0.31206 & & & & & & -0.427297891 & & & \\
\hline PRDM1 & & -0.36333 & & & & -0.3515 & & & & & & \\
\hline PRELP & & & -0.32754 & & & & & & & & & -0.514571263 \\
\hline CPQ & & & -0.46067 & -0.49325 & & & & & & & & \\
\hline DDX3X & -0.28456 & & & & -0.28196 & & & & & & & \\
\hline CLEC11A & & & -0.34123 & -0.46276 & & & & & & & & \\
\hline HLA-DQA2 & -0.79236 & & & & & & & -0.95838 & & & & \\
\hline ANXA1 & & & & & & & & -0.40609 & & & & -0.537935229 \\
\hline LTBP4 & & & -0.8311 & -0.45264 & & & & & & & & \\
\hline ACKR3 & & & -0.50398 & -0.42836 & & & & & & & & \\
\hline GSTP1 & & & & & & & -0.44941 & & & & -0.51106 & \\
\hline CKB & & & & & & & -0.28237 & & & & -0.85408 & \\
\hline TNXB & & & -0.88375 & -0.37198 & & & & & & & & \\
\hline COL5A1 & & & -0.32296 & -0.25878 & & & & & & & & \\
\hline LPAR6 & & -0.4566 & & & & -0.462 & & & & & & \\
\hline FBLN2 & & & -0.45008 & -0.35248 & & & & & & & & \\
\hline CRABP2 & & & & -0.33398 & & & & & & & -1.25347 & \\
\hline SPARCL1 & -0.40323 & & -1.08891 & & & & & & & & & \\
\hline AP1S2 & & & & & & & & & & & -1.37364 & -0.585902618 \\
\hline TUBB2A & & & -0.30543 & & & & & & & & -2.14209 & \\
\hline MAML2 & & & -0.29751 & & & & & & -0.403869121 & & & \\
\hline TUBA1B & & -0.30403 & & & & & & & & & -0.75739 & \\
\hline PCSK1N & & & & -0.4453 & & & & & & & -2.40024 & \\
\hline KRT10 & & & & -0.26069 & & & -0.82497 & & & & & \\
\hline ANGPTL1 & & & -0.9719 & -0.67573 & & & & & & & & \\
\hline STC1 & -0.80954 & & & & & & & & & -0.8037 & & \\
\hline ABCC9 & & & -0.34722 & -0.30007 & & & & & & & & \\
\hline PHF20 & & & & & & -0.32896 & & & -0.376242178 & & & \\
\hline ANKRD28 & & -0.39239 & & & & & & -0.3962 & & & & \\
\hline ADRB2 & -0.36268 & & & & & & -0.38184 & & & & & \\
\hline IL6ST & & -0.30985 & & & -0.25819 & & & & & & & \\
\hline UBB & & -0.30301 & & & & -0.2718 & & & & & & \\
\hline HSPB1 & & & -0.33181 & & & & & -0.73136 & & & & \\
\hline CLK1 & & & -0.26105 & & & -0.27463 & & & & & & \\
\hline Sox4 & & & -0.28575 & & & & & & & & -1.79864 & \\
\hline DEFB1 & & & & -0.45588 & & & -0.45578 & & & & & \\
\hline CES1 & & & -0.32475 & -0.4616 & & & & & & & & \\
\hline FBLN1 & & & -0.77964 & -0.47717 & & & & & & & & \\
\hline CCDC80 & & & -0.90537 & -0.67581 & & & & & & & & \\
\hline DUSP1 & & -0.468 & & & & & & -0.35648 & & & & \\
\hline CCPG1 & & & -0.29446 & -0.29595 & & & & & & & & \\
\hline GOLIM4 & & & -0.37095 & -0.29703 & & & & & & & & \\
\hline DDIT4 & & & -0.29078 & & & -0.3101 & & & & & & \\
\hline CASC4 & & & -0.28677 & -0.28713 & & & & & & & & \\
\hline OMD & & & -0.35084 & -0.61405 & & & & & & & & \\
\hline DNAJA1 & -0.30245 & & & & -0.34791 & & & & & & & \\
\hline LEPR & & & -0.72288 & -0.40868 & & & & & & & & \\
\hline
\end{tabular}




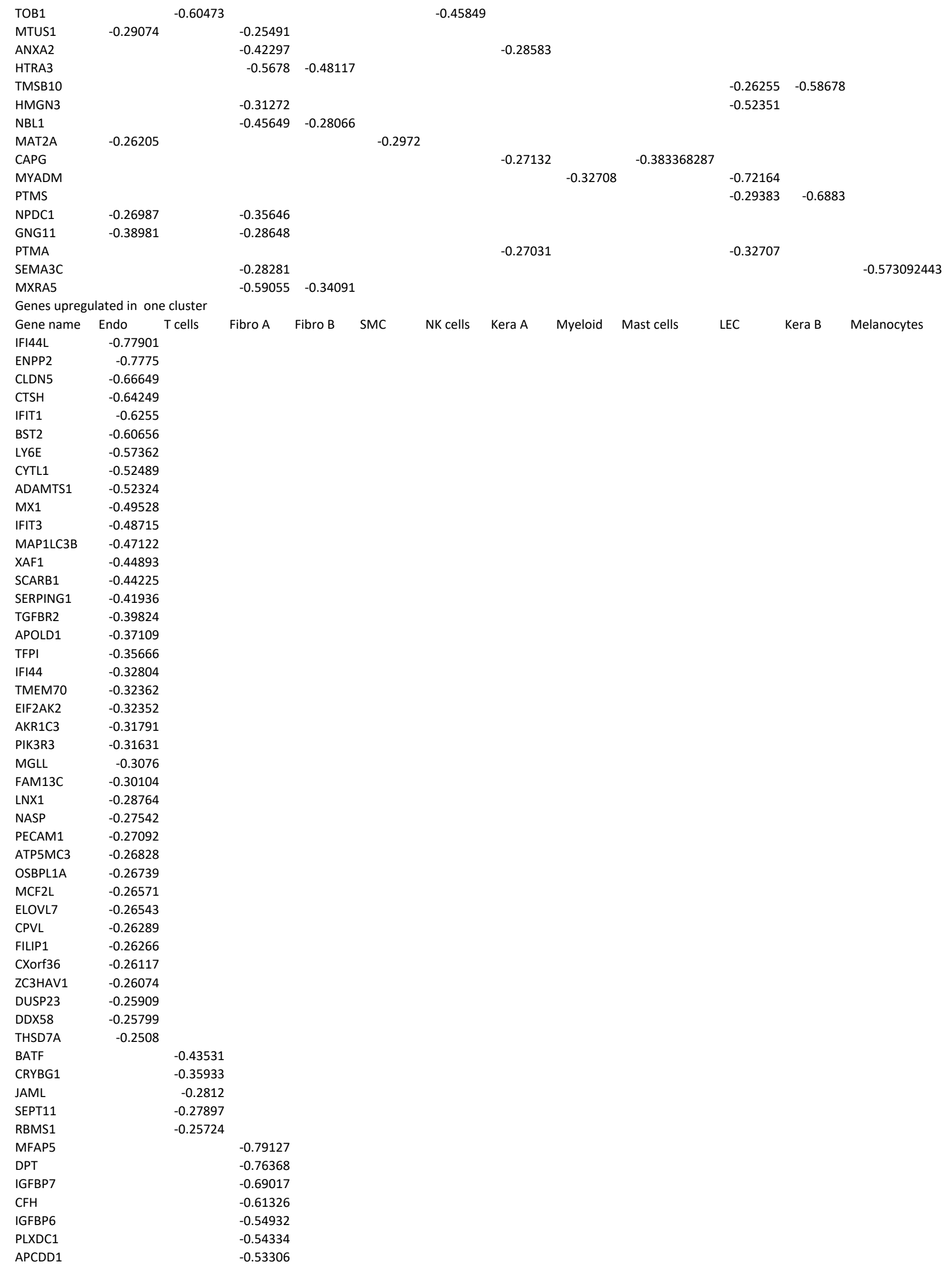




\begin{tabular}{|c|c|}
\hline CLEC3B & -0.52001 \\
\hline SDC2 & -0.5091 \\
\hline TRPS1 & -0.48686 \\
\hline ZBTB20 & -0.47576 \\
\hline SVIL & -0.4733 \\
\hline DPYSL3 & -0.46593 \\
\hline ELN & -0.46114 \\
\hline ABI3BP & -0.4509 \\
\hline SPTBN1 & -0.44321 \\
\hline TACC1 & -0.42466 \\
\hline MXRA7 & -0.41706 \\
\hline SH3BGRL3 & -0.41605 \\
\hline ABCA8 & -0.40272 \\
\hline FBN1 & -0.40108 \\
\hline OLFML2B & -0.38761 \\
\hline PTN & -0.38567 \\
\hline SYNE1 & -0.37273 \\
\hline CCDC186 & -0.37008 \\
\hline PLEKHH2 & -0.36765 \\
\hline CD34 & -0.36723 \\
\hline TMOD3 & -0.36315 \\
\hline ANTXR1 & -0.36254 \\
\hline ARL6IP5 & -0.36077 \\
\hline PSD3 & -0.34215 \\
\hline AXL & -0.33391 \\
\hline GAS7 & -0.33347 \\
\hline NFIA & -0.3334 \\
\hline TIMP3 & -0.33318 \\
\hline IL16 & -0.33286 \\
\hline LUM & -0.32643 \\
\hline LTBP3 & -0.32597 \\
\hline EPB41L2 & -0.31327 \\
\hline ANK2 & -0.3125 \\
\hline BMP4 & -0.31015 \\
\hline DYNC2LI1 & -0.30811 \\
\hline $\mathrm{DIO} 2$ & -0.30769 \\
\hline COLEC12 & -0.30127 \\
\hline WEE1 & -0.2971 \\
\hline GADD45G & -0.29478 \\
\hline CTNNB1 & -0.2905 \\
\hline $\mathrm{F} 2 \mathrm{R}$ & -0.28972 \\
\hline ID3 & -0.28833 \\
\hline ZNF706 & -0.28674 \\
\hline EMP3 & -0.28652 \\
\hline PMEPA1 & -0.28646 \\
\hline PER2 & -0.28374 \\
\hline TUBB4B & -0.28366 \\
\hline COL5A2 & -0.28327 \\
\hline HNRNPDL & -0.28303 \\
\hline NFIX & -0.28281 \\
\hline OSR1 & -0.28029 \\
\hline BLOC1S1 & -0.2798 \\
\hline TMEM43 & -0.27755 \\
\hline EIF4A2 & -0.27735 \\
\hline LIMCH1 & -0.2766 \\
\hline PGRMC1 & -0.27617 \\
\hline PREX2 & -0.27514 \\
\hline PALLD & -0.27352 \\
\hline KLF4 & -0.27307 \\
\hline MAN1A1 & -0.27199 \\
\hline NORAD & -0.27099 \\
\hline CTSF & -0.27035 \\
\hline DNM1 & -0.27031 \\
\hline SRSF2 & -0.26797 \\
\hline RBM6 & -0.26786 \\
\hline ARGLU1 & -0.26706 \\
\hline PAM & -0.26644 \\
\hline WWTR1 & -0.26595 \\
\hline МОВзВ & -0.26591 \\
\hline
\end{tabular}




\begin{tabular}{|c|c|c|c|}
\hline SEPT7 & -0.26523 & & \\
\hline CAMK1D & -0.26395 & & \\
\hline RGS10 & -0.26395 & & \\
\hline ADAM33 & -0.26251 & & \\
\hline REEP5 & -0.26204 & & \\
\hline ECM1 & -0.26172 & & \\
\hline C9orf3 & -0.26007 & & \\
\hline SNHG7 & -0.25969 & & \\
\hline DDAH2 & -0.25691 & & \\
\hline GPSM2 & -0.25567 & & \\
\hline GLDN & -0.25464 & & \\
\hline ADD3 & -0.2524 & & \\
\hline MEOX2 & -0.25235 & & \\
\hline IRS2 & -0.25217 & & \\
\hline SNRPN & -0.25144 & & \\
\hline ID1 & -0.25005 & & \\
\hline SLPI & & -1.54995 & \\
\hline C1QTNF3 & & -0.76975 & \\
\hline MATN4 & & -0.67267 & \\
\hline ITIH5 & & -0.61034 & \\
\hline LRRC15 & & -0.54122 & \\
\hline MFGE8 & & -0.49319 & \\
\hline CERCAM & & -0.47858 & \\
\hline NUCB2 & & -0.46318 & \\
\hline FNDC1 & & -0.45069 & \\
\hline FAP & & -0.44733 & \\
\hline SEMA3B & & -0.44044 & \\
\hline ANGPTL5 & & -0.43049 & \\
\hline PPIC & & -0.40564 & \\
\hline CYBRD1 & & -0.37934 & \\
\hline VKORC1 & & -0.37742 & \\
\hline P4HA2 & & -0.36184 & \\
\hline FAM180B & & -0.3486 & \\
\hline PCOLCE2 & & -0.34208 & \\
\hline PCOLCE & & -0.34179 & \\
\hline$X G$ & & -0.33538 & \\
\hline CITED2 & & -0.32734 & \\
\hline ADGRA2 & & -0.32559 & \\
\hline PRDX4 & & -0.30593 & \\
\hline HAS2 & & -0.30591 & \\
\hline FKBP7 & & -0.30113 & \\
\hline KDELR3 & & -0.29378 & \\
\hline C1QTNF7 & & -0.28753 & \\
\hline PDGFRL & & -0.28664 & \\
\hline SKAP2 & & -0.28316 & \\
\hline OGN & & -0.28086 & \\
\hline C1QTNF4 & & -0.28043 & \\
\hline AlF1L & & -0.27517 & \\
\hline CHPF & & -0.27172 & \\
\hline OSR2 & & -0.26525 & \\
\hline GSTM5 & & -0.26352 & \\
\hline SERPINA5 & & -0.26222 & \\
\hline LRRC17 & & -0.26198 & \\
\hline MMP27 & & -0.26054 & \\
\hline RECK & & -0.25322 & \\
\hline CCL8 & & & -0.8383 \\
\hline $\mathrm{CH} 25 \mathrm{H}$ & & & -0.60632 \\
\hline AVPR1A & & & -0.59526 \\
\hline RGS5 & & & -0.53366 \\
\hline MYC & & & -0.49778 \\
\hline NCKAP5 & & & -0.49622 \\
\hline ANGPT2 & & & -0.48011 \\
\hline MTHFD2 & & & -0.46523 \\
\hline C2orf40 & & & -0.43381 \\
\hline CLMN & & & -0.40702 \\
\hline ZNF331 & & & -0.3769 \\
\hline ANGPTL4 & & & -0.33705 \\
\hline ADRA2A & & & -0.32314 \\
\hline SNHG15 & & & -0.31405 \\
\hline
\end{tabular}




\begin{tabular}{|c|c|c|c|}
\hline COX412 & -0.30969 & & \\
\hline ENPEP & -0.30347 & & \\
\hline GGCX & -0.28732 & & \\
\hline LBH & -0.28328 & & \\
\hline MEF2C & -0.28233 & & \\
\hline NUDT4 & -0.28219 & & \\
\hline RRAD & -0.27053 & & \\
\hline MCL1 & -0.26835 & & \\
\hline KLHL23 & -0.2658 & & \\
\hline СYTH3 & -0.25329 & & \\
\hline MT1F & & -1.41651 & \\
\hline AREG & & -0.65314 & \\
\hline SLC30A1 & & -0.48577 & \\
\hline FGFBP2 & & -0.37768 & \\
\hline CMC1 & & -0.32381 & \\
\hline GNLY & & -0.32011 & \\
\hline ISG20 & & -0.28525 & \\
\hline SKI & & -0.28444 & \\
\hline DEDD2 & & -0.28386 & \\
\hline LINC01871 & & -0.28314 & \\
\hline RPL27 & & -0.27703 & \\
\hline ATP5IF1 & & -0.25786 & \\
\hline ELL2 & & -0.25664 & \\
\hline GLRX & & -0.25527 & \\
\hline LGALS7 & & & -1.45927 \\
\hline KRT1 & & & -0.92113 \\
\hline MT1G & & & -0.88288 \\
\hline KRTDAP & & & -0.86028 \\
\hline HOPX & & & -0.71286 \\
\hline DMKN & & & -0.70337 \\
\hline LY6D & & & -0.66791 \\
\hline CALML5 & & & -0.64173 \\
\hline SYT8 & & & -0.61808 \\
\hline MT4 & & & -0.61555 \\
\hline CCL27 & & & -0.58423 \\
\hline HBA2 & & & -0.5643 \\
\hline DSG1 & & & -0.55309 \\
\hline EPHB6 & & & -0.55033 \\
\hline KCNK7 & & & -0.54356 \\
\hline PHLDA2 & & & -0.53725 \\
\hline LGALS7B & & & -0.53064 \\
\hline DSP & & & -0.52483 \\
\hline CSTA & & & -0.52185 \\
\hline SBSN & & & -0.49566 \\
\hline DSC1 & & & -0.49261 \\
\hline S100A14 & & & -0.49127 \\
\hline PYCARD & & & -0.49041 \\
\hline ALDH3A2 & & & -0.48217 \\
\hline ALDH3A1 & & & -0.4708 \\
\hline AHNAK2 & & & -0.46498 \\
\hline IL18 & & & -0.43455 \\
\hline CYB5A & & & -0.43232 \\
\hline CCND1 & & & -0.42449 \\
\hline $\mathrm{RNH1}$ & & & -0.41843 \\
\hline S100A16 & & & -0.41199 \\
\hline GLTP & & & -0.40769 \\
\hline PKP1 & & & -0.39295 \\
\hline LAMTOR4 & & & -0.37774 \\
\hline SDC1 & & & -0.37387 \\
\hline EMP2 & & & -0.37155 \\
\hline RAB25 & & & -0.37154 \\
\hline CLTB & & & -0.36399 \\
\hline JUP & & & -0.35814 \\
\hline WNT4 & & & -0.34775 \\
\hline CHP2 & & & -0.34042 \\
\hline CASP14 & & & -0.32774 \\
\hline EPPK1 & & & -0.3274 \\
\hline CLDN1 & & & -0.32406 \\
\hline LGALSL & & & -0.31102 \\
\hline
\end{tabular}




\begin{tabular}{|c|c|}
\hline TRIM29 & -0.29809 \\
\hline ZBTB7B & -0.29716 \\
\hline OGFRL1 & -0.29375 \\
\hline FGFBP1 & -0.29348 \\
\hline SULT2B1 & -0.29249 \\
\hline CEBPA & -0.29109 \\
\hline IMPA2 & -0.29104 \\
\hline SYTL1 & -0.28551 \\
\hline DSC3 & -0.28278 \\
\hline PKP3 & -0.28147 \\
\hline EGR3 & -0.28104 \\
\hline PPP1R16A & -0.27649 \\
\hline LYPD3 & -0.27438 \\
\hline ANO1 & -0.2737 \\
\hline CD109 & -0.2728 \\
\hline ITM2B & -0.27137 \\
\hline EIF2S3 & -0.26555 \\
\hline DUSP2 & -0.26479 \\
\hline TKT & -0.26012 \\
\hline KLK8 & -0.2588 \\
\hline CAMSAP3 & -0.2583 \\
\hline HBEGF & -0.25662 \\
\hline KLF13 & -0.25501 \\
\hline MGST2 & -0.25304 \\
\hline CAPNS2 & -0.25174 \\
\hline CLCA4 & -0.25033 \\
\hline \multicolumn{2}{|l|}{ IL1R2 } \\
\hline \multicolumn{2}{|l|}{ FCER1A } \\
\hline \multicolumn{2}{|l|}{ LGALS2 } \\
\hline \multicolumn{2}{|l|}{ CD1C } \\
\hline \multicolumn{2}{|l|}{ S100A8 } \\
\hline \multicolumn{2}{|l|}{ ARL4C } \\
\hline \multicolumn{2}{|l|}{ CD1E } \\
\hline \multicolumn{2}{|l|}{ PELI1 } \\
\hline \multicolumn{2}{|l|}{ CD37 } \\
\hline \multicolumn{2}{|l|}{ P2RY14 } \\
\hline \multicolumn{2}{|l|}{ FCGR2B } \\
\hline \multicolumn{2}{|l|}{ S100A9 } \\
\hline \multicolumn{2}{|l|}{ MIS18BP1 } \\
\hline \multicolumn{2}{|l|}{ CDC42EP3 } \\
\hline RARA & \\
\hline CFP & \\
\hline SMAP2 & \\
\hline SGMS2 & \\
\hline FAU & \\
\hline SYTL3 & \\
\hline HEY1 & \\
\hline ALOX5 & \\
\hline HIST1H4C & \\
\hline IL18R1 & \\
\hline FDX1 & \\
\hline SLC26A2 & \\
\hline PIK3R1 & \\
\hline IKZF1 & \\
\hline CPEB4 & \\
\hline ABCA1 & \\
\hline ITK & \\
\hline STK4 & \\
\hline AHNAK & \\
\hline DUSP4 & \\
\hline IL1RL1 & \\
\hline VPS37B & \\
\hline JARID2 & \\
\hline MAN2A1 & \\
\hline LRRFIP1 & \\
\hline MAPK1 & \\
\hline HERC1 & \\
\hline FYN & \\
\hline FAM102B & \\
\hline
\end{tabular}


CAV1

$-1.04784$

ADAMTS6

$-1.01894$

NFATC2

$-0.72301$

TCTN3

$-0.70816$

SCN3A

$-0.54455$

CALM1

$-0.54427$

PLPP1

$-0.5122$

ARHGAP10

$-0.51197$

GUCY1A1

$-0.49014$

$-0.45569$

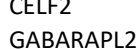

$-0.3666$

HINT1

TUBA1A

$-0.35967$

TUBB2B

STMN2

RBP1

HOXB5

MLLT11

GAP43

DCX

MAP1B

RTN1

BEX1

HOTAIRM1

JPT1

UCHL1

NREP

GNG3

NEFL

BEX2

BEX3

NSG1

LINC02381

TAGLN3

TCEAL7

LAMP5

NEFM

SCG5

KIF5C

C4orf48

ZFHX3

SOX11

SNCG

HOXA5

STMN4

TMSB15A

MDK

CRMP1

SST

PAFAH1B3

FXYD6

VAMP2

HOXB8

INA

CCNI

ELAVL4

ATP6VOB

POU2F2

CRABP1

CENPV

NCAM1

HOXB2

YBX1

FEZ1

$\mathrm{CNIH} 2$

APLP1

ZNF428

OLFM1

MAP1LC3A

$-3.73683$

$-3.25627$

$-2.90726$

$-2.64275$

$-2.60621$

$-2.24695$

$-2.24496$

$-2.2171$

$-2.20634$

$-2.128$

$-2.07028$

$-1.96144$

$-1.94581$

$-1.88067$

$-1.79246$

$-1.75608$

$-1.75137$

$-1.71223$

$-1.67834$

$-1.67748$

$-1.61979$

$-1.58756$

$-1.56335$

$-1.51583$

$-1.49855$

$-1.45784$

$-1.43897$

$-1.40574$

$-1.38517$

$-1.38513$

$-1.3714$

$-1.3391$

$-1.32065$

$-1.28789$

$-1.27723$

$-1.22141$

$-1.21481$

$-1.20885$

$-1.20113$

$-1.18005$

$-1.16459$

$-1.16248$

$-1.14434$

$-1.13919$

$-1.1325$

$-1.12679$

$-1.11066$

$-1.10941$

$-1.09197$

$-1.08041$

$-1.04755$

$-1.03924$

$-1.03864$

$-1.02574$

$-1.01801$

$-1.0138$

$-0.98545$ 
PKIA

$-0.93618$

SVBP

$-0.92974$

UBE2E3

$-0.92035$

PFN2

RBFOX2

$-0.89671$

$-0.879$

NBDY

$-0.87747$

CXXC5

$-0.82716$

MYL6B

$-0.82229$

YWHAQ

$-0.81009$

PSMB5

$-0.80279$

RPAIN

$-0.79811$

PHPT1

$-0.78173$

MAGED1

$-0.76154$

YWHAB

$-0.65876$

GNAS

NDUFC2

$-0.60519$

$-0.57445$

$-0.56248$

$-0.53049$

H3F3A

SUMO2

$-0.51854$

$-0.41907$

CD24

$-0.38941$

GPNMB

SORBS2

DCT

$-0.684110075$

$-0.60254962$

$-0.530983028$

ITGB1

$-0.37777787$ 


\begin{tabular}{|c|c|c|c|c|c|c|c|c|c|c|c|c|}
\hline Gene name & Endo & T cells & Fibro A & Fibro B & SMC & NK cells & Kera A & Myeloid & Mast cells & LEC & Kera B & Melanocytes \\
\hline NME2 & 0.547258 & 0.371458 & 0.340721 & 0.363589 & 0.418803 & 0.331886 & 0.450537 & 0.6064299 & 0.56595293 & 0.730945 & 1.094215 & 0.623357197 \\
\hline RPS4Y1 & 0.703169 & 0.435065 & 1.174829 & 0.69737 & 0.585449 & & 1.084121 & 0.5619834 & 0.41518773 & 0.396801 & 0.80169 & \\
\hline HLA-C & 0.378065 & 0.498066 & & & 0.527427 & 0.448201 & & 0.5733192 & 0.42208544 & 0.564683 & 0.97714 & 0.874216762 \\
\hline MIF & & 0.324424 & 0.42757 & 0.457057 & 0.437317 & 0.295345 & & 0.5113932 & 0.40276355 & & 1.623008 & 0.704981498 \\
\hline MT-ND3 & 0.37092 & 0.336789 & & & 0.546428 & 0.251835 & 0.374305 & 0.2582309 & 0.3412849 & 0.466307 & & \\
\hline HLA-A & & 0.484634 & & 0.384055 & & 0.337589 & & 0.5563169 & & 0.478607 & 0.583014 & 0.595157841 \\
\hline DDX3Y & & 0.395287 & 0.271111 & 0.302641 & & & 0.934603 & 0.4582154 & & 0.328329 & 0.368335 & \\
\hline RPL23 & 0.431415 & & 0.86344 & 0.560244 & 0.320165 & & 0.341373 & & 0.36003773 & & 0.301384 & \\
\hline RPS20 & 0.298897 & 0.310132 & 1.274441 & 0.716998 & & 0.314092 & & 0.2527761 & 0.56255778 & & & \\
\hline RBM3 & 0.299571 & & 0.503899 & 0.390994 & 0.365508 & 0.250225 & 0.275376 & 0.283415 & & & & \\
\hline RPS29 & 0.331461 & & 0.746293 & 0.445427 & & & 0.476095 & & 0.33143794 & & 0.592135 & \\
\hline IRF1 & & 0.336207 & 0.534242 & 0.527859 & & 0.352049 & 0.422992 & & & & 0.793662 & \\
\hline RPL37 & 0.323441 & & 0.671545 & 0.443982 & & & 0.2677 & & 0.34457422 & & 0.625977 & \\
\hline RPL21 & 0.258341 & & 0.579715 & 0.323685 & & & & & 0.49470926 & & 0.345427 & \\
\hline RPL38 & & & 0.698942 & 0.420935 & & & 0.396723 & & 0.30367766 & & 0.419501 & \\
\hline TPT1 & 0.3115 & & 0.587283 & 0.333613 & & & & & 0.37142071 & & 0.433872 & \\
\hline RPL37A & 0.262739 & & 0.669676 & 0.381003 & & & 0.273572 & & & & 0.495042 & \\
\hline CXCL2 & 0.679417 & & 0.70651 & 0.957998 & & & & 0.3073845 & & 0.658737 & & \\
\hline IER3 & 0.342594 & & & 0.980697 & 0.352337 & & 0.275186 & & & & 1.263731 & \\
\hline RPS11 & 0.39486 & & 0.70314 & 0.482959 & & & 0.358009 & & 0.3774103 & & & \\
\hline RPS27 & 0.251796 & & 0.604392 & 0.397959 & & & & & 0.34503906 & & 0.577146 & \\
\hline RPS6 & 0.316809 & & 0.699359 & 0.452469 & & & & & 0.47683545 & & 0.320883 & \\
\hline IFITM1 & & 0.702658 & & & 0.288515 & 0.481955 & & & & & 0.753352 & 0.578707739 \\
\hline RPL13A & 0.275171 & & 1.033401 & 0.571162 & & & 0.319765 & & 0.53549743 & & & \\
\hline SNHG8 & & & 0.337829 & 0.331813 & & & 0.350569 & & 0.26660765 & & 0.728808 & \\
\hline PTGS2 & 0.422843 & & 0.254272 & & & & & 0.5086329 & 0.47199476 & & & \\
\hline NAMPT & & 0.348548 & 0.388493 & 0.27208 & & & & & & & 0.694073 & \\
\hline RPL23A & & & 0.491178 & 0.272827 & & & & & 0.26729174 & & 0.427923 & \\
\hline RPS25 & & & 0.531156 & 0.310336 & & & & & 0.30070986 & & 0.399008 & \\
\hline BHLHE40 & & 0.338586 & & 0.272905 & & & 0.410202 & & & & 0.766516 & \\
\hline EML4 & & 0.305545 & & & & 0.353217 & 0.282952 & & & & 0.432537 & \\
\hline $\mathrm{SLC} 2 \mathrm{~A} 3$ & & 0.558417 & & 0.453869 & & 0.303038 & & 0.2962175 & & & & \\
\hline RPS2 & 0.26518 & & 0.857408 & 0.462609 & & & & & 0.26783415 & & & \\
\hline ETV6 & & 0.317364 & & & & & & 0.2585767 & & 0.386445 & 0.592726 & \\
\hline SOD2 & & & 0.384681 & 0.507807 & & & & 0.3138256 & & & 1.157378 & \\
\hline TAGLN & 0.807621 & & & 0.740199 & 0.504397 & & 0.340915 & & & & & \\
\hline RPS14 & 0.306783 & & 0.538675 & 0.369955 & & & & & 0.3453059 & & & \\
\hline RPL34 & & & 0.389567 & 0.280926 & & & & & 0.25143096 & & 0.267645 & \\
\hline RPLP2 & & & 0.734354 & 0.317802 & & & & & 0.34491503 & & 0.375578 & \\
\hline HLA-E & & & & 0.424987 & & & 0.27148 & 0.2637002 & & & 0.586215 & \\
\hline IFI16 & & & 0.337891 & & & & 0.263973 & & 0.50000352 & & 0.509849 & \\
\hline LDLR & & & 0.437886 & 0.456628 & & & 0.324238 & & & & & \\
\hline RPL17 & & & 0.32456 & & & & & & 0.465309 & & 0.799049 & \\
\hline COL4A2 & & & 0.253665 & 0.530039 & 0.478362 & & & & & & & \\
\hline RPL7 & & & 0.791679 & 0.561236 & & & & & 0.31577267 & & & \\
\hline NAP1L1 & & & 0.252867 & 0.275305 & & & & & 0.42463917 & & & \\
\hline RPL10A & & & 0.508807 & 0.351754 & & & & & 0.3538282 & & & \\
\hline CD44 & & & & & 0.269347 & & 0.319974 & & & & 0.78241 & \\
\hline ARL5B & & 0.336951 & & & & & 0.386872 & 0.3554569 & & & & \\
\hline RPL35 & & & 0.517336 & 0.266194 & & & & & & & 0.363259 & \\
\hline RPSA & & & 0.57613 & 0.28499 & & & & & 0.31555735 & & & \\
\hline RPL8 & & & 0.55653 & 0.292155 & & & & & & & & 0.413216758 \\
\hline HSPB6 & & & 0.672439 & 0.312341 & 0.303763 & & & & & & & \\
\hline RPL19 & & & 0.409251 & 0.250473 & & & & & & & 0.296089 & \\
\hline CREM & & 0.447252 & & & & & & 0.3770373 & 0.45069937 & & & \\
\hline NOP53 & & & 0.431115 & 0.275035 & & & & & & & 0.383433 & \\
\hline RPL31 & & & 0.747204 & 0.383637 & & & & & 0.37556957 & & & \\
\hline $\mathrm{RHOH}$ & & 0.337693 & & & & 0.276707 & & & & & 0.386867 & \\
\hline RPS18 & & & 0.56113 & 0.290567 & & & & & & & 0.396979 & \\
\hline
\end{tabular}




\begin{tabular}{|c|c|c|c|c|c|c|c|c|c|c|c|c|}
\hline GZMA & & 0.897744 & & & & 0.535586 & & & & & 1.005085 & \\
\hline PLAU & & & 0.394493 & 0.65853 & 0.313711 & & & & & & & \\
\hline UBA52 & & & 0.472994 & 0.276849 & & & & & & & 0.410045 & \\
\hline PTPN22 & & 0.27491 & & & & 0.336918 & & & & & 0.278387 & \\
\hline MT-ATP6 & 0.275827 & & & & 0.411076 & & 0.361411 & & & & & \\
\hline EIF1AY & & & & & & & 0.288129 & & & 0.289001 & 0.28376 & \\
\hline RPL39 & & & 0.616353 & 0.345179 & & & & & & & 0.446785 & \\
\hline MYL9 & 0.329095 & & & 0.262138 & 0.329908 & & & & & & & \\
\hline REL & & 0.436042 & & & & 0.36065 & 0.572132 & & & & & \\
\hline PTX3 & & & 1.651101 & 1.499872 & & & & & & 0.919685 & & \\
\hline LDHA & & & & & & & 0.364783 & 0.2641583 & & & 1.537097 & \\
\hline VMP1 & & 0.342952 & & & & & & 0.3119368 & & 0.256952 & & \\
\hline CHCHD10 & & & 0.293929 & & & & 0.272511 & & & & 0.982745 & \\
\hline RPL13 & & & 0.541552 & 0.287425 & & & & & 0.28618076 & & & \\
\hline RNASEK & & & & & & & & & 0.42043937 & 0.350011 & 0.591158 & \\
\hline NNMT & & & 0.507078 & 0.412303 & & & & & & 0.377083 & & \\
\hline NFKBIA & & & & 0.38601 & & & 0.641001 & & & & 0.905982 & \\
\hline ACTN1 & 0.367638 & & & & 0.409057 & & 0.363334 & & & & & \\
\hline RPS12 & & & 0.376859 & 0.27668 & & & & & & & 0.297525 & \\
\hline RPL3 & 0.318354 & & 0.272569 & & & & & & & & 0.273176 & \\
\hline JUND & & & & & & 0.257835 & 0.547616 & & & & 0.467872 & \\
\hline PNRC1 & & & 0.280984 & 0.408034 & & & & & & & 0.692512 & \\
\hline NR4A2 & & 0.461788 & & & & 0.30024 & & & & & 0.5413 & \\
\hline PSME1 & & 0.291917 & & & & & & & 0.32598938 & & 0.372933 & \\
\hline RPLP1 & & & 0.448969 & 0.334658 & & & & & & & 0.384178 & \\
\hline POSTN & 0.40625 & & & & 1.438816 & & 0.802632 & & & & & \\
\hline SAMSN1 & & 0.465959 & & & & 0.293526 & & & & & 0.507746 & \\
\hline IGFBP4 & & & 0.485642 & 0.313848 & & & & & & 0.497847 & & \\
\hline CTSL & & & 0.544402 & 0.360061 & & & & 0.3782209 & & & & \\
\hline RPL14 & & & 0.456347 & 0.276712 & & & & & & & 0.303823 & \\
\hline SPOCK1 & & & 0.276063 & 0.495204 & & & & & & & & \\
\hline NDUFA13 & & & & & & & & & 0.31425909 & & 0.573849 & \\
\hline VGLL3 & & & & 0.481054 & 0.423996 & & & & & & & \\
\hline $\mathrm{A} 2 \mathrm{M}$ & & & & & 0.256286 & & & 0.4303081 & & & & \\
\hline AC013394.1 & & & & & & & 0.255506 & & & & 0.490509 & \\
\hline ADAMTS4 & & & 0.390886 & & & & 0.293001 & & & & & \\
\hline RPS3A & & & 0.368547 & 0.264685 & & & & & & & & \\
\hline JUN & & & & & & & 0.330505 & & & & 0.41144 & \\
\hline AEBP1 & & & & & 0.302246 & & & & & 0.340027 & & \\
\hline RPS8 & & & 0.540369 & 0.333604 & & & & & & & & \\
\hline PPP1R15A & & & & 0.348592 & & & 0.316006 & & & & & \\
\hline $\mathrm{VCL}$ & 0.253096 & & & & 0.351652 & & & & & & & \\
\hline CCNL1 & & & & & & & 0.262115 & & & & 0.423104 & \\
\hline CLEC2B & & 0.495874 & & & & & & & & & 0.614586 & \\
\hline PRDX1 & & & & & & & & 0.3296437 & & & 0.330863 & \\
\hline CYTOR & & & 0.393616 & 0.540826 & & & & & & & & \\
\hline SLMAP & & & & & 0.348139 & & 0.277009 & & & & & \\
\hline IGF1 & & & 0.630271 & 0.61299 & & & & & & & & \\
\hline GBP5 & & 0.364517 & & & & 0.336078 & & & & & & \\
\hline PDCD4 & & & & & & 0.267624 & & & & & 0.885823 & \\
\hline RPL24 & & & 0.361126 & 0.260698 & & & & & & & & \\
\hline STK17A & & 0.271381 & & & & & & & & & 0.400868 & \\
\hline GPR183 & & 0.320569 & & & & 0.396337 & & & & & & \\
\hline JUNB & & & & 0.325956 & & & & & & & 0.726252 & \\
\hline RPL18 & & & 0.499374 & 0.27386 & & & & & & & & \\
\hline TRAF1 & & 0.309719 & & & & 0.31794 & & & & & & \\
\hline KLF2 & & 0.334431 & & & 0.2857 & & & & & & & \\
\hline WISP2 & & & 0.324839 & & 0.495733 & & & & & & & \\
\hline GZMK & & 0.939808 & & & & 0.739561 & & & & & & \\
\hline TM4SF1 & & & & & 0.411118 & & & & & & 0.665745 & \\
\hline THBS1 & 0.275996 & & & 0.288923 & & & & & & & & \\
\hline NPC2 & & & 0.305122 & & & & & 0.4620318 & & & & \\
\hline FOS & & & & 0.345056 & & & & & & & 0.702445 & \\
\hline RPL18A & & & 0.500667 & 0.268323 & & & & & & & & \\
\hline МYСВР2 & & & 0.296078 & & & 0.258893 & & & & & & \\
\hline PDIA3 & & & & & & & & 0.401891 & 0.30815955 & & & \\
\hline RPL36 & & & 0.546304 & & & & & & & & 0.359688 & \\
\hline LAP3 & & & & & & & & 0.4306416 & & & 0.380079 & \\
\hline CYBA & & & & & & & & 0.3556592 & & & & 0.469762734 \\
\hline PSMB9 & & & & & & 0.307234 & & & & & 0.361761 & \\
\hline
\end{tabular}




\begin{tabular}{|c|c|c|c|c|c|c|c|c|c|c|c|}
\hline PFN1 & & 0.272738 & & & 0.345763 & & & & & & \\
\hline EHF & & & & & & 0.474491 & & & & 0.518376 & \\
\hline HLA-DPA1 & & & & & 0.328866 & & & & & 0.410757 & \\
\hline COL4A1 & & 0.279346 & 0.455606 & & & & & & & & \\
\hline RPLPO & & 0.683061 & 0.464984 & & & & & & & & \\
\hline RPS15 & & 0.263445 & & & & & & & & 0.33028 & \\
\hline CCL5 & & 0.561283 & & & & & & & & 0.621615 & \\
\hline C11orf96 & 0.277611 & & 0.706518 & & & & & & & & \\
\hline TC2N & & 0.319878 & & & & & & & & 0.419653 & \\
\hline CTSC & & & 0.279138 & & & & 0.5484772 & & & & \\
\hline EIF3E & & 0.374512 & 0.26759 & & & & & & & & \\
\hline TNFAIP2 & & 0.851235 & 0.718327 & & & & & & & & \\
\hline GMFG & & & & & & & & & & 0.324652 & 0.837565566 \\
\hline IFNG & & 0.839134 & & & 0.289834 & & & & & & \\
\hline GBP1 & & & 0.300263 & & & & 0.4085794 & & & & \\
\hline ACTA2 & & & 0.300453 & 0.532373 & & & & & & & \\
\hline CSRP1 & & & & 0.548255 & & & & & & 0.798639 & \\
\hline RPL32 & & 0.415709 & 0.295845 & & & & & & & & \\
\hline ARID5B & & & 0.27048 & & & 0.333978 & & & & & \\
\hline CNN2 & & 0.275073 & 0.279846 & & & & & & & & \\
\hline ADM & & 0.406561 & 0.644805 & & & & & & & & \\
\hline STAT3 & & 0.259635 & & & & & & & & 0.416988 & \\
\hline ROMO1 & & & & & & & & 0.33996868 & & 0.660254 & \\
\hline HLA-DPB1 & & & & & 0.318478 & & & & & 0.287081 & \\
\hline MYLK & & 0.250545 & & 0.374301 & & & & & & & \\
\hline RPS28 & & 0.360071 & & & & & & & & 0.520279 & \\
\hline CD59 & & & & & & & 0.3292268 & & & 0.522039 & \\
\hline SAT1 & & & & & & 0.2628 & & & & 0.777452 & \\
\hline CHD2 & & 0.274143 & & & & 0.368611 & & & & & \\
\hline TMEM176B & & & & & & & 0.3413587 & 0.55228924 & & & \\
\hline TGFBR3 & & & & & & & & & 0.401072 & 0.324872 & \\
\hline INSIG1 & & 0.41338 & 0.396899 & & & & & & & & \\
\hline RPL9 & & 0.467529 & & & & & & 0.27614554 & & & \\
\hline LTB & & 0.756876 & & & 0.425952 & & & & & & \\
\hline HEBP2 & & 0.279315 & & & & & & & & 0.417105 & \\
\hline PSME2 & & & & & 0.291203 & & 0.3263267 & & & & \\
\hline BICDL1 & & 0.422531 & & & 0.334875 & & & & & & \\
\hline RPS16 & & 0.683385 & 0.452265 & & & & & & & & \\
\hline GADD45B & & & & & & 0.532568 & & & & 1.366078 & \\
\hline TNFAIP8 & & & & & & & 0.3365882 & & & 0.59883 & \\
\hline BCL2A1 & & 0.308391 & & & & & 0.4275594 & & & & \\
\hline CAV2 & 0.274651 & & & 0.296048 & & & & & & & \\
\hline SRPX & & 0.408348 & 0.269481 & & & & & & & & \\
\hline SFRP1 & & & 0.557206 & & & & & & & 1.226404 & \\
\hline RNF213 & & 0.337043 & & & 0.263026 & & & & & & \\
\hline SVEP1 & & 0.916465 & 0.86663 & & & & & & & & \\
\hline WSB1 & & & & & & 0.363833 & & & 0.415543 & & \\
\hline CD3D & & 0.299257 & & & 0.390531 & & & & & & \\
\hline GPRIN3 & & 0.310183 & & & 0.289713 & & & & & & \\
\hline SERPINE1 & 0.371671 & & 0.492716 & & & & & & & & \\
\hline ACTN4 & 0.29228 & & & & & & & & & 0.366836 & \\
\hline KRT14 & 0.268832 & & & 0.502033 & & & & & & & \\
\hline CALD1 & 0.562582 & & & & & 0.254955 & & & & & \\
\hline HIF1A & & & 0.375585 & & & & & & & 0.631932 & \\
\hline RPL12 & & 0.526198 & 0.30719 & & & & & & & & \\
\hline COX7B & & & & & & & & 0.27791528 & & 0.722407 & \\
\hline PARP14 & & & & & 0.316424 & & & & 0.359175 & & \\
\hline BTF3 & & 0.262837 & 0.256902 & & & & & & & & \\
\hline GADD45A & & & 0.409258 & & & 0.48649 & & & & & \\
\hline CST7 & & 0.519616 & & & & & & 0.30082726 & & & \\
\hline NFKB1 & & 0.26643 & & & & & & & & 0.409123 & \\
\hline RPL35A & & 0.33898 & 0.254596 & & & & & & & & \\
\hline ACKR4 & & 0.257395 & 0.484332 & & & & & & & & \\
\hline ICAM1 & & 0.336523 & & & & & 0.3166565 & & & & \\
\hline CA2 & & & & & & 0.485681 & & & & 0.601311 & \\
\hline DHRS3 & & 0.309625 & 0.460217 & & & & & & & & \\
\hline RASGRP1 & & 0.388064 & & & 0.335621 & & & & & & \\
\hline LINC00861 & & 0.526087 & & & 0.290645 & & & & & & \\
\hline IER2 & & & & 0.407373 & & & & & & 0.584492 & \\
\hline CCL14 & 0.425375 & & & & & & & & 0.823013 & & \\
\hline
\end{tabular}




\begin{tabular}{|c|c|c|c|c|c|c|c|c|c|c|c|c|}
\hline $\mathrm{ADH} 1 \mathrm{~B}$ & & & 0.406369 & 0.278142 & & & & & & & & \\
\hline NKTR & & & & & & & 0.260651 & & & 0.368015 & & \\
\hline TNC & & & & 0.898797 & 0.500155 & & & & & & & \\
\hline PPIF & & & & & & & & 0.3367992 & & & 0.372163 & \\
\hline MYDGF & & & & & & & & 0.291494 & 0.33996122 & & & \\
\hline CXCL12 & 0.462423 & & 0.260863 & & & & & & & & & \\
\hline TIMP1 & 0.269986 & & & & & & & & & 0.913106 & & \\
\hline GPX3 & & & 0.790299 & 0.262428 & & & & & & & & \\
\hline CD36 & & & 0.654819 & & & & & 0.374781 & & & & \\
\hline IL32 & & 0.442842 & & & & 0.322425 & & & & & & \\
\hline C6orf48 & & & 0.309802 & 0.308411 & & & & & & & & \\
\hline NFE2L2 & & 0.409511 & & & & & & & & & 0.443756 & \\
\hline ID4 & & & & 0.526073 & & & & & & & 0.813289 & \\
\hline DDX21 & & 0.465893 & & & & 0.370762 & & & & & & \\
\hline CLSTN2 & & & 0.29142 & & 0.306228 & & & & & & & \\
\hline LYST & & & & & & 0.360405 & & & & 0.351299 & & \\
\hline BIRC3 & & & & & & 0.294856 & & & & & 0.718774 & \\
\hline GZMM & & 0.392151 & & & & & & & & & 0.349853 & \\
\hline RPS9 & & & 0.272342 & & & & & & & & 0.288411 & \\
\hline EIF4A3 & & & & 0.258956 & & & 0.274192 & & & & & \\
\hline SELENOP & & & & & & & & 0.5706181 & & & 0.422864 & \\
\hline PCAT19 & 0.361504 & & & & & & & & & 0.366629 & & \\
\hline NINJ1 & & & & & & & & 0.3606283 & & & 0.606782 & \\
\hline TAPBP & & 0.284843 & & & & & & & & & 0.345004 & \\
\hline EEF1A1 & & & 0.300873 & 0.365649 & & & & & & & & \\
\hline CLEC2D & & 0.321342 & & & & 0.371981 & & & & & & \\
\hline RPL29 & & & 0.528192 & 0.330467 & & & & & & & & \\
\hline BRD2 & & & & & & & 0.367719 & & & & 0.439061 & \\
\hline GPC3 & & & 0.471761 & 1.012248 & & & & & & & & \\
\hline KRT15 & & & & & & & 0.567353 & & & & 0.84945 & \\
\hline PTPRC & & 0.291106 & & & & 0.252117 & & & & & & \\
\hline GAPDH & & & & & 0.318953 & & & & & & 0.813968 & \\
\hline CD48 & & & & & & & & & 0.31442918 & & 0.323214 & \\
\hline LIMD2 & & 0.325097 & & & & 0.309887 & & & & & & \\
\hline RPS13 & & & 0.376202 & 0.253051 & & & & & & & & \\
\hline SFN & & & & & 0.275099 & & & & & & 0.802554 & \\
\hline RPS5 & & & 0.541218 & 0.343905 & & & & & & & & \\
\hline \multicolumn{13}{|c|}{ Genes upregulated in one cluster } \\
\hline Gene name & Endo & T cells & Fibro A & Fibro B & SMC & NK cells & Kera A & Myeloid & Mast cells & LEC & Kera B & Melanocytes \\
\hline TCIM & 0.465767 & & & & & & & & & & & \\
\hline CPE & 0.436767 & & & & & & & & & & & \\
\hline CX3CL1 & 0.393712 & & & & & & & & & & & \\
\hline MMRN1 & 0.385103 & & & & & & & & & & & \\
\hline CTNNAL1 & 0.328307 & & & & & & & & & & & \\
\hline HSPG2 & 0.315073 & & & & & & & & & & & \\
\hline NUAK1 & 0.313232 & & & & & & & & & & & \\
\hline PLS3 & 0.310562 & & & & & & & & & & & \\
\hline STK38L & 0.28956 & & & & & & & & & & & \\
\hline CXCL8 & 0.288281 & & & & & & & & & & & \\
\hline RAI14 & 0.288279 & & & & & & & & & & & \\
\hline SLCO2A1 & 0.286451 & & & & & & & & & & & \\
\hline SAMD4A & 0.268979 & & & & & & & & & & & \\
\hline YWHAH & 0.264533 & & & & & & & & & & & \\
\hline SMDT1 & 0.264185 & & & & & & & & & & & \\
\hline RGPD2 & & 0.510405 & & & & & & & & & & \\
\hline ICOS & & 0.385625 & & & & & & & & & & \\
\hline CTLA4 & & 0.383268 & & & & & & & & & & \\
\hline JAK3 & & 0.365574 & & & & & & & & & & \\
\hline GIMAP7 & & 0.360547 & & & & & & & & & & \\
\hline SATB1 & & 0.341232 & & & & & & & & & & \\
\hline AP3M2 & & 0.336155 & & & & & & & & & & \\
\hline CD2 & & 0.296482 & & & & & & & & & & \\
\hline ANKRD12 & & 0.289405 & & & & & & & & & & \\
\hline USP36 & & 0.289386 & & & & & & & & & & \\
\hline AC004687.1 & & 0.288034 & & & & & & & & & & \\
\hline IL2RB & & 0.281947 & & & & & & & & & & \\
\hline P2RY8 & & 0.27314 & & & & & & & & & & \\
\hline CHD1 & & 0.272356 & & & & & & & & & & \\
\hline CD7 & & 0.27213 & & & & & & & & & & \\
\hline ZC3H12D & & 0.27161 & & & & & & & & & & \\
\hline
\end{tabular}




\begin{tabular}{|c|c|c|}
\hline UTY & 0.269459 & \\
\hline SPOCK2 & 0.262779 & \\
\hline TAGAP & 0.262534 & \\
\hline GPR65 & 0.258413 & \\
\hline RIPOR2 & 0.256638 & \\
\hline PDE4D & 0.256082 & \\
\hline PDE3B & 0.255277 & \\
\hline HNRNPA3 & 0.25395 & \\
\hline KDM6B & 0.25197 & \\
\hline TNFAIP6 & 0.877525 & \\
\hline C7 & 0.791587 & \\
\hline CPXM1 & 0.712987 & \\
\hline MGST1 & 0.514455 & \\
\hline LPL & 0.501426 & \\
\hline APOC1 & 0.482055 & \\
\hline DEPP1 & 0.454422 & \\
\hline RPL11 & 0.424697 & \\
\hline VCAM1 & 0.418938 & \\
\hline MTURN & 0.394376 & \\
\hline RPL7A & 0.380168 & \\
\hline AGTR1 & 0.37598 & \\
\hline RPL6 & 0.374854 & \\
\hline SMOC1 & 0.371412 & \\
\hline RPS3 & 0.355467 & \\
\hline IMPDH2 & 0.351149 & \\
\hline RPL4 & 0.349913 & \\
\hline EPB41L4A-AS1 & 0.348938 & \\
\hline EEF1B2 & 0.345334 & \\
\hline SNHG19 & 0.343044 & \\
\hline PLXNA4 & 0.34185 & \\
\hline RARRES2 & 0.329936 & \\
\hline RPL22 & 0.323292 & \\
\hline ALKAL2 & 0.321779 & \\
\hline PQLC2L & 0.319932 & \\
\hline RPS24 & 0.318466 & \\
\hline APOL6 & 0.316065 & \\
\hline MAP7D3 & 0.314175 & \\
\hline RPS7 & 0.303037 & \\
\hline RBPMS & 0.302562 & \\
\hline NACA & 0.300366 & \\
\hline RPS15A & 0.298825 & \\
\hline G0S2 & 0.288391 & \\
\hline EEF2 & 0.285902 & \\
\hline CPB1 & 0.283511 & \\
\hline ZFHX4 & 0.27295 & \\
\hline RPS27A & 0.263828 & \\
\hline RPS23 & 0.263653 & \\
\hline PTGDS & 0.257411 & \\
\hline $\mathrm{FBL}$ & 0.255708 & \\
\hline TLN1 & 0.254251 & \\
\hline CDKN1C & 0.251287 & \\
\hline COMP & & 1.447902 \\
\hline LTBP2 & & 0.655553 \\
\hline GEM & & 0.543237 \\
\hline SERPINE2 & & 0.532598 \\
\hline CDH11 & & 0.522386 \\
\hline COL12A1 & & 0.514896 \\
\hline EFEMP1 & & 0.512167 \\
\hline PLA2G2A & & 0.495771 \\
\hline SMOC2 & & 0.477769 \\
\hline WTAP & & 0.476296 \\
\hline PDLIM2 & & 0.433081 \\
\hline F3 & & 0.421106 \\
\hline LAMC1 & & 0.406313 \\
\hline COL8A1 & & 0.399905 \\
\hline TNNT3 & & 0.39606 \\
\hline MYOC & & 0.394293 \\
\hline ABL2 & & 0.384691 \\
\hline GGT5 & & 0.379511 \\
\hline NFIB & & 0.379319 \\
\hline
\end{tabular}




\begin{tabular}{|c|c|}
\hline C3 & 0.354788 \\
\hline CYGB & 0.354622 \\
\hline RARRES1 & 0.34104 \\
\hline AMOTL2 & 0.339903 \\
\hline ATP2B1 & 0.339349 \\
\hline PDGFD & 0.338747 \\
\hline LRRC32 & 0.335232 \\
\hline TMEM119 & 0.322778 \\
\hline UGCG & 0.318439 \\
\hline ZFAS1 & 0.314725 \\
\hline RGS16 & 0.314031 \\
\hline SCARA3 & 0.313795 \\
\hline MYL12A & 0.310499 \\
\hline NAV1 & 0.301395 \\
\hline TNFRSF12A & 0.29945 \\
\hline ZYX & 0.296976 \\
\hline COL18A1 & 0.294335 \\
\hline PTGES & 0.291212 \\
\hline NTRK2 & 0.288353 \\
\hline GPRC5B & 0.284707 \\
\hline MAFF & 0.283525 \\
\hline ATP5MC2 & 0.27328 \\
\hline WNT11 & 0.27197 \\
\hline TSHZ2 & 0.266991 \\
\hline CRLF1 & 0.265112 \\
\hline PRRX1 & 0.264371 \\
\hline CMTM3 & 0.262849 \\
\hline PPL & 0.260844 \\
\hline LSP1 & 0.2561 \\
\hline GLIS3 & 0.255112 \\
\hline RFLNB & 0.252095 \\
\hline PXDC1 & 0.251499 \\
\hline ACTG2 & 0.632645 \\
\hline COL6A1 & 0.625939 \\
\hline PDLIM5 & 0.49334 \\
\hline FGF7 & 0.490262 \\
\hline SYNPO2 & 0.486753 \\
\hline CNN1 & 0.448996 \\
\hline FHL5 & 0.434962 \\
\hline PRUNE2 & 0.425823 \\
\hline SGIP1 & 0.423203 \\
\hline MYH11 & 0.413502 \\
\hline TPM2 & 0.35496 \\
\hline LTBP1 & 0.341307 \\
\hline CARMN & 0.324451 \\
\hline MACF1 & 0.322664 \\
\hline PDLIM7 & 0.318012 \\
\hline C12orf75 & 0.311507 \\
\hline FLNA & 0.311237 \\
\hline IGFBP2 & 0.30698 \\
\hline MBNL1-AS1 & 0.305196 \\
\hline MYH9 & 0.298964 \\
\hline MRVI1 & 0.285004 \\
\hline TGFB1/1 & 0.284948 \\
\hline CYFIP2 & 0.283232 \\
\hline RTN4 & 0.283077 \\
\hline SELENOM & 0.282691 \\
\hline KANK2 & 0.279914 \\
\hline PELI2 & 0.275164 \\
\hline CAVIN3 & 0.273091 \\
\hline MT-ND5 & 0.271591 \\
\hline EDIL3 & 0.27158 \\
\hline ITGA1 & 0.268201 \\
\hline LPP & 0.264585 \\
\hline BAG2 & 0.259004 \\
\hline CAVIN1 & 0.258587 \\
\hline CCDC3 & 0.258497 \\
\hline SORBS1 & 0.258155 \\
\hline FNBP1 & 0.256671 \\
\hline YAP1 & 0.251955 \\
\hline
\end{tabular}




\begin{tabular}{|c|c|c|}
\hline CRTAM & 0.834386 & \\
\hline XCL1 & 0.571033 & \\
\hline COTL1 & 0.459571 & \\
\hline KLRG1 & 0.447474 & \\
\hline TIGIT & 0.425214 & \\
\hline GZMH & 0.400324 & \\
\hline HLA-DQB1 & 0.384285 & \\
\hline HLA-DQA1 & 0.364956 & \\
\hline CD8A & 0.360668 & \\
\hline CORO1A & 0.342673 & \\
\hline RNF19A & 0.338507 & \\
\hline SH2D1A & 0.325815 & \\
\hline APBB1IP & 0.318829 & \\
\hline AC093010.2 & 0.31716 & \\
\hline PYHIN1 & 0.311271 & \\
\hline EVI2A & 0.304879 & \\
\hline IKZF3 & 0.298219 & \\
\hline FYB1 & 0.294577 & \\
\hline NR4A3 & 0.292708 & \\
\hline LEPROTL1 & 0.281207 & \\
\hline TNFSF9 & 0.273999 & \\
\hline CD28 & 0.270644 & \\
\hline GPR174 & 0.269773 & \\
\hline ENC1 & 0.268165 & \\
\hline RBM25 & 0.265457 & \\
\hline CD3G & 0.265418 & \\
\hline PNN & 0.255125 & \\
\hline DTHD1 & 0.254007 & \\
\hline SLAMF7 & 0.250412 & \\
\hline FABP7 & & 1.123206 \\
\hline EGOT & & 0.727649 \\
\hline PMAIP1 & & 0.689865 \\
\hline NSUN6 & & 0.657849 \\
\hline INTS6 & & 0.639419 \\
\hline CACNB4 & & 0.638455 \\
\hline SMG1 & & 0.581258 \\
\hline KRT6A & & 0.580473 \\
\hline ARL4A & & 0.538373 \\
\hline ZNF217 & & 0.531921 \\
\hline NEDD4L & & 0.517672 \\
\hline LINC00842 & & 0.491947 \\
\hline GSDMC & & 0.465095 \\
\hline AL355075.4 & & 0.44885 \\
\hline GABPB1-AS1 & & 0.448614 \\
\hline MALAT1 & & 0.439545 \\
\hline IRF6 & & 0.439053 \\
\hline EZH2 & & 0.436119 \\
\hline TSC22D2 & & 0.416517 \\
\hline TIAM1 & & 0.405989 \\
\hline SDCCAG8 & & 0.397749 \\
\hline AHCYL1 & & 0.391278 \\
\hline SEMA4A & & 0.385975 \\
\hline SLC5A3 & & 0.38486 \\
\hline ARIH1 & & 0.382903 \\
\hline HNRNPU & & 0.381054 \\
\hline FBXW7 & & 0.3798966 \\
\hline ITGA2 & & 0.379652 \\
\hline DSC2 & & 0.377179 \\
\hline IDI1 & & 0.376592 \\
\hline CENPL & & 0.375005 \\
\hline AC007952.4 & & 0.371697 \\
\hline IFRD1 & & 0.369869 \\
\hline TXNRD1 & & 0.361263 \\
\hline CXADR & & 0.36007 \\
\hline MUCL1 & & 0.358907 \\
\hline SERPINB7 & & 0.357405 \\
\hline EIF4A1 & & 0.354774 \\
\hline EPHA2 & & 0.351158 \\
\hline ABI1 & & 0.344765 \\
\hline TRIM69 & & 0.343214 \\
\hline
\end{tabular}




\begin{tabular}{|c|c|c|}
\hline DENND2C & 0.34164 & \\
\hline PCF11 & 0.339796 & \\
\hline RND3 & 0.339632 & \\
\hline ERF & 0.334248 & \\
\hline GOLGA4 & 0.331544 & \\
\hline MSN & 0.330485 & \\
\hline MFHAS1 & 0.323394 & \\
\hline FAT1 & 0.321481 & \\
\hline ASXL1 & 0.320056 & \\
\hline SPPL3 & 0.319911 & \\
\hline GOLGA8A & 0.319306 & \\
\hline TRA2B & 0.319204 & \\
\hline NFAT5 & 0.317398 & \\
\hline PPP1R10 & 0.317366 & \\
\hline TRA2A & 0.312161 & \\
\hline KDSR & 0.311642 & \\
\hline SQSTM1 & 0.306498 & \\
\hline YME1L1 & 0.300408 & \\
\hline C3orf52 & 0.297495 & \\
\hline MRPS6 & 0.290138 & \\
\hline RB1CC1 & 0.2867 & \\
\hline TEAD1 & 0.282553 & \\
\hline POLR2A & 0.282428 & \\
\hline MAP7D1 & 0.282098 & \\
\hline MED13L & 0.281615 & \\
\hline SERTAD2 & 0.280575 & \\
\hline AC103591.3 & 0.279301 & \\
\hline TNFRSF19 & 0.279198 & \\
\hline ETFDH & 0.278992 & \\
\hline IVNS1ABP & 0.278546 & \\
\hline HCAR3 & 0.275554 & \\
\hline SMURF2 & 0.274444 & \\
\hline LUC7L3 & 0.274165 & \\
\hline GCH1 & 0.271938 & \\
\hline FUS & 0.267854 & \\
\hline RRAGC & 0.267809 & \\
\hline DENND4A & 0.267687 & \\
\hline MORC3 & 0.266964 & \\
\hline PDK1 & 0.264906 & \\
\hline BAZ1A & 0.261646 & \\
\hline TRAK1 & 0.25872 & \\
\hline SRSF3 & 0.258401 & \\
\hline WAC & 0.258287 & \\
\hline NPIPB5 & 0.257915 & \\
\hline RNF152 & 0.257876 & \\
\hline GOLGB1 & 0.25689 & \\
\hline ASH1L & 0.255744 & \\
\hline PDGFC & 0.253286 & \\
\hline SERBP1 & 0.251402 & \\
\hline CCL13 & & 1.0271155 \\
\hline FCGR3A & & 0.9967379 \\
\hline $\mathrm{C} 1 \mathrm{QB}$ & & 0.981146 \\
\hline PLTP & & 0.9652652 \\
\hline FCGBP & & 0.923438 \\
\hline RNASE1 & & 0.8606108 \\
\hline F13A1 & & 0.8142813 \\
\hline IL1RN & & 0.7918724 \\
\hline CCL3 & & 0.7208858 \\
\hline CD14 & & 0.7110873 \\
\hline CTSB & & 0.6923458 \\
\hline CCL4 & & 0.6630761 \\
\hline CCL3L1 & & 0.6542435 \\
\hline CD209 & & 0.5853251 \\
\hline C1QA & & 0.5794059 \\
\hline C1QC & & 0.5777074 \\
\hline IL1B & & 0.5582776 \\
\hline LYVE1 & & 0.5385784 \\
\hline CYBB & & 0.4997182 \\
\hline MS4A4A & & 0.4843311 \\
\hline ADAP2 & & 0.4533922 \\
\hline
\end{tabular}




\begin{tabular}{|c|}
\hline PLAUR \\
\hline SELENOK \\
\hline CR1 \\
\hline MARCO \\
\hline DAB2 \\
\hline LGMN \\
\hline PDIA6 \\
\hline IL4I1 \\
\hline SLCO2B1 \\
\hline CD163 \\
\hline FCGR1A \\
\hline HSPA5 \\
\hline HSP9OB1 \\
\hline FOLR2 \\
\hline TTYH3 \\
\hline PLEK \\
\hline CTSZ \\
\hline SDF2L1 \\
\hline FCN1 \\
\hline DRAM1 \\
\hline CALR \\
\hline CD93 \\
\hline LAIR1 \\
\hline GK \\
\hline MAF \\
\hline CALM3 \\
\hline C2 \\
\hline CD63 \\
\hline CREG1 \\
\hline CLTC \\
\hline ITGB2 \\
\hline MANF \\
\hline PDIA4 \\
\hline MSR1 \\
\hline ARPC1B \\
\hline ADAM9 \\
\hline PSAP \\
\hline AP1B1 \\
\hline CYFIP1 \\
\hline TMED9 \\
\hline LAMP1 \\
\hline BLVRB \\
\hline SNX6 \\
\hline PPIB \\
\hline MIR155HG \\
\hline SLC15A3 \\
\hline LILRB1 \\
\hline ATF5 \\
\hline MLEC \\
\hline FAM198B \\
\hline EGR2 \\
\hline P4HB \\
\hline DSE \\
\hline GPR34 \\
\hline CLTA \\
\hline IGSF6 \\
\hline NAIP \\
\hline SPINT2 \\
\hline CANX \\
\hline CD4 \\
\hline KCTD12 \\
\hline CCR1 \\
\hline RPN2 \\
\hline STAT1 \\
\hline POMP \\
\hline SDC3 \\
\hline FUCA1 \\
\hline LRP1 \\
\hline ITSN1 \\
\hline LIPA \\
\hline
\end{tabular}

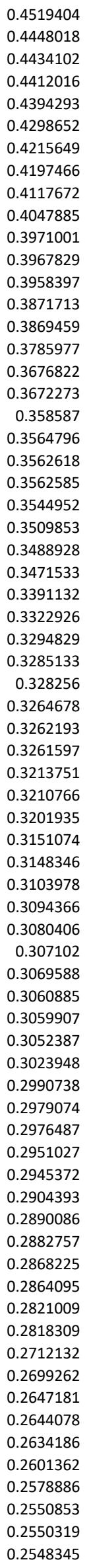




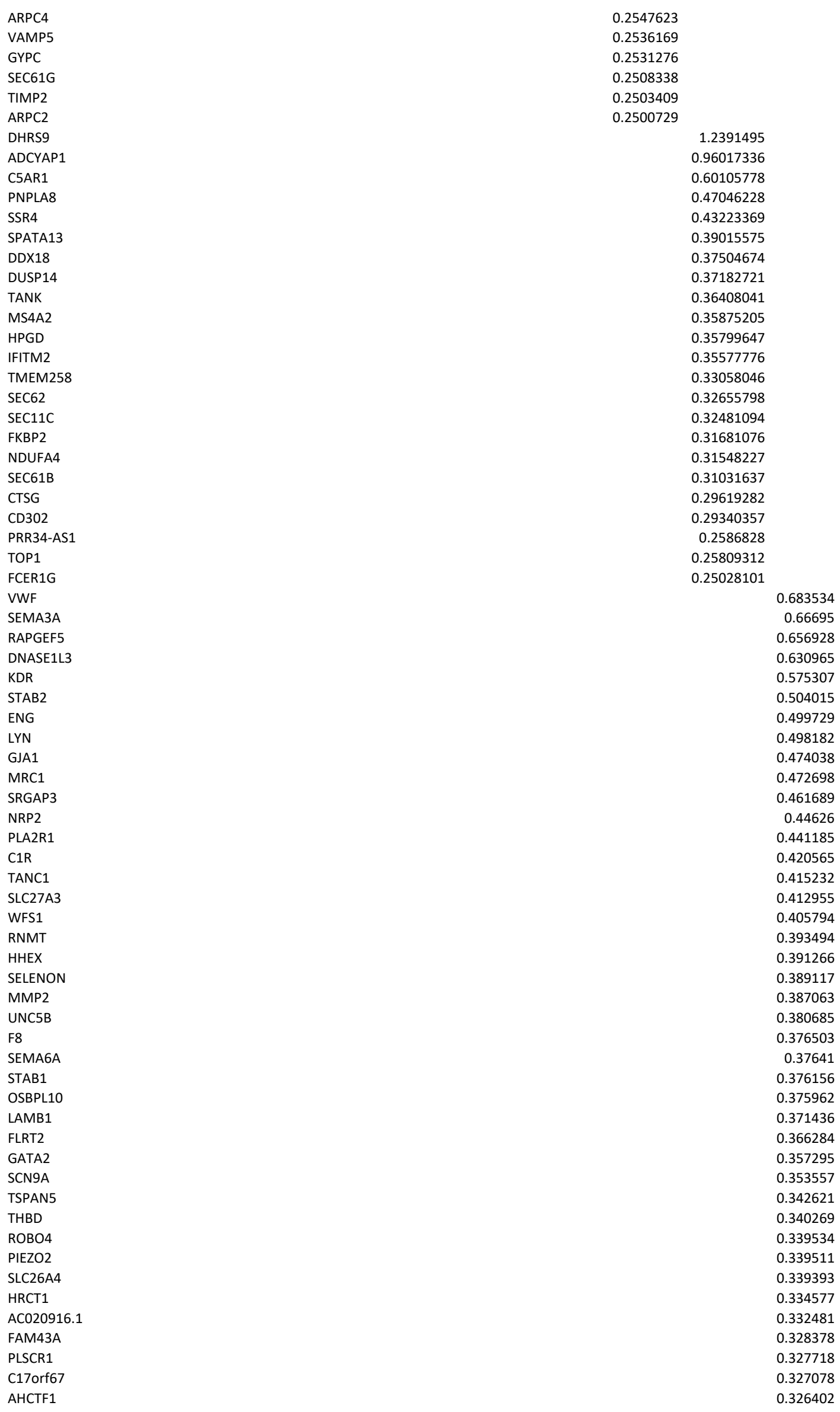




\begin{tabular}{|c|c|c|}
\hline TMEM255B & 0.322463 & \\
\hline ANKRD29 & 0.320182 & \\
\hline SHC1 & 0.319984 & \\
\hline PRR5L & 0.318192 & \\
\hline KLHL3 & 0.311179 & \\
\hline GPR146 & 0.308527 & \\
\hline NPR1 & 0.303095 & \\
\hline RHOU & 0.299152 & \\
\hline PLIN5 & 0.297235 & \\
\hline MFAP2 & 0.292658 & \\
\hline QSOX1 & 0.292233 & \\
\hline MCFD2 & 0.29115 & \\
\hline TXNL4B & 0.289699 & \\
\hline IL7 & 0.285137 & \\
\hline SPTLC2 & 0.284392 & \\
\hline RALGAPA2 & 0.283335 & \\
\hline PDE7B & 0.281847 & \\
\hline ETS2 & 0.278823 & \\
\hline RARRES3 & 0.278242 & \\
\hline CASP4 & 0.275003 & \\
\hline YTHDC2 & 0.273383 & \\
\hline CFI & 0.273318 & \\
\hline TNRC6B & 0.272653 & \\
\hline BCAT1 & 0.269073 & \\
\hline MMP14 & 0.267636 & \\
\hline CARD16 & 0.265131 & \\
\hline DDR2 & 0.26161 & \\
\hline TSPAN11 & 0.254784 & \\
\hline AQP5 & & 1.697469 \\
\hline AKR1C2 & & 1.638407 \\
\hline TPD52L1 & & 1.181765 \\
\hline PPP1R1B & & 1.161149 \\
\hline FXYD3 & & 1.160622 \\
\hline S100A1 & & 1.120962 \\
\hline TESC & & 1.077989 \\
\hline CA6 & & 1.072322 \\
\hline KRT19 & & 1.045407 \\
\hline SPTSSB & & 1.005262 \\
\hline VAMP8 & & 0.972985 \\
\hline NDUFB1 & & 0.964817 \\
\hline ZG16B & & 0.939881 \\
\hline S100A11 & & 0.91309 \\
\hline KRT18 & & 0.900977 \\
\hline SNORC & & 0.900841 \\
\hline PDLIM4 & & 0.900314 \\
\hline KRT7 & & 0.887937 \\
\hline POLR2L & & 0.880846 \\
\hline CLDN10 & & 0.873833 \\
\hline NDRG2 & & 0.868332 \\
\hline CEBPB & & 0.865683 \\
\hline SLC12A2 & & 0.862772 \\
\hline RHOV & & 0.85969 \\
\hline NDUFA1 & & 0.781371 \\
\hline ANAPC16 & & 0.774329 \\
\hline ALDH9A1 & & 0.772797 \\
\hline TMEM123 & & 0.768317 \\
\hline KRT8 & & 0.758676 \\
\hline STAC2 & & 0.755513 \\
\hline COX7A1 & & 0.754076 \\
\hline ELF3 & & 0.751995 \\
\hline SLC25A5 & & 0.710218 \\
\hline REXO2 & & 0.702138 \\
\hline COX5B & & 0.694464 \\
\hline TSTD1 & & 0.682516 \\
\hline ADI1 & & 0.678753 \\
\hline TOMM7 & & 0.667447 \\
\hline NDUFC1 & & 0.626678 \\
\hline ENO1 & & 0.624397 \\
\hline CRACR2B & & 0.623329 \\
\hline GCHFR & & 0.620646 \\
\hline
\end{tabular}




\begin{tabular}{|c|c|}
\hline ROPN1B & 0.61634 \\
\hline ACTR3B & 0.604301 \\
\hline PALMD & 0.599997 \\
\hline CAST & 0.598908 \\
\hline FAM162A & 0.592113 \\
\hline SDCBP & 0.590408 \\
\hline PDE4B & 0.572509 \\
\hline UQCR11 & 0.562418 \\
\hline SDHD & 0.557871 \\
\hline MTIF3 & 0.55695 \\
\hline NDUFB4 & 0.550423 \\
\hline ETHE1 & 0.550296 \\
\hline NIPSNAP2 & 0.548197 \\
\hline H2AFJ & 0.540965 \\
\hline ANKRD37 & 0.540241 \\
\hline PPA1 & 0.538863 \\
\hline GSTK1 & 0.533828 \\
\hline CHPT1 & 0.522906 \\
\hline UQCRQ & 0.513964 \\
\hline UGP2 & 0.508121 \\
\hline ECHDC2 & 0.505171 \\
\hline CMPK1 & 0.504991 \\
\hline ARHGDIB & 0.503094 \\
\hline AES & 0.501275 \\
\hline GNG5 & 0.499631 \\
\hline cox14 & 0.496154 \\
\hline cox17 & 0.494857 \\
\hline SMIM22 & 0.48548 \\
\hline PIM3 & 0.485165 \\
\hline OSTF1 & 0.482391 \\
\hline BTG2 & 0.48111 \\
\hline ATP5F1B & 0.480267 \\
\hline EEF1D & 0.472095 \\
\hline $\operatorname{cox} 7 \mathrm{C}$ & 0.469291 \\
\hline CYSTM1 & 0.468357 \\
\hline SH3YL1 & 0.46767 \\
\hline ABHD5 & 0.465675 \\
\hline GLS & 0.464564 \\
\hline WDR45B & 0.464528 \\
\hline ALDOA & 0.460889 \\
\hline TPD52 & 0.458181 \\
\hline WASF2 & 0.458164 \\
\hline CDC42SE2 & 0.455533 \\
\hline PGK1 & 0.455175 \\
\hline NDUFA3 & 0.454988 \\
\hline COX6B1 & 0.453129 \\
\hline DNAJC1 & 0.45205 \\
\hline SPTSSA & 0.45068 \\
\hline DECR1 & 0.450119 \\
\hline ARFGAP3 & 0.444157 \\
\hline MKNK2 & 0.443384 \\
\hline ETF1 & 0.441294 \\
\hline NDUFB2 & 0.441199 \\
\hline LMO4 & 0.440659 \\
\hline TOMM20 & 0.440313 \\
\hline ATP5MG & 0.440205 \\
\hline TMEM141 & 0.439445 \\
\hline CEMIP2 & 0.43941 \\
\hline PHB & 0.438831 \\
\hline HADHB & 0.436345 \\
\hline CNN3 & 0.435105 \\
\hline CD46 & 0.432156 \\
\hline UQCRB & 0.429588 \\
\hline SRRM1 & 0.425347 \\
\hline PSMB8 & 0.42526 \\
\hline TPI1 & 0.422865 \\
\hline SSFA2 & 0.422631 \\
\hline SYPL1 & 0.422098 \\
\hline RORA & 0.421573 \\
\hline EZR & 0.420509 \\
\hline
\end{tabular}




\begin{tabular}{|c|c|}
\hline MBD2 & 0.419389 \\
\hline VDAC1 & 0.419042 \\
\hline IQGAP2 & 0.419033 \\
\hline ClB1 & 0.416005 \\
\hline CLIC1 & 0.415643 \\
\hline ANP32B & 0.414728 \\
\hline RHOC & 0.409709 \\
\hline LITAF & 0.409355 \\
\hline NANS & 0.409262 \\
\hline ESRRA & 0.409005 \\
\hline SGK1 & 0.408992 \\
\hline UBL5 & 0.408239 \\
\hline ARHGAP18 & 0.407033 \\
\hline SUCLA2 & 0.402934 \\
\hline NUDT8 & 0.402921 \\
\hline ANXA11 & 0.402378 \\
\hline ATP5ME & 0.400316 \\
\hline HADHA & 0.400026 \\
\hline ANXA5 & 0.398151 \\
\hline SMIM4 & 0.394784 \\
\hline ISCU & 0.392663 \\
\hline PKM & 0.388094 \\
\hline COX8A & 0.388078 \\
\hline SYF2 & 0.387324 \\
\hline UBALD2 & 0.386048 \\
\hline WIPF1 & 0.385897 \\
\hline GRPEL1 & 0.38589 \\
\hline CD164 & 0.383805 \\
\hline UBE2V1 & 0.383498 \\
\hline ATP5MD & 0.382701 \\
\hline CFLAR & 0.381894 \\
\hline МАР2К3 & 0.381763 \\
\hline SERP1 & 0.380266 \\
\hline EIF3K & 0.379847 \\
\hline SRSF5 & 0.379297 \\
\hline TMEM159 & 0.375304 \\
\hline ATP5MF & 0.374509 \\
\hline STK39 & 0.368824 \\
\hline SLC9A3R1 & 0.368065 \\
\hline ZFP36L1 & 0.36505 \\
\hline APOBECЗG & 0.361623 \\
\hline CDC37 & 0.359247 \\
\hline ID2 & 0.357712 \\
\hline МАРЗК8 & 0.357429 \\
\hline PNP & 0.356378 \\
\hline ARID5A & 0.35381 \\
\hline CHMP4B & 0.351181 \\
\hline HLA-F & 0.350443 \\
\hline ANP32E & 0.350346 \\
\hline TUBA4A & 0.347847 \\
\hline PPP2R5A & 0.345525 \\
\hline NENF & 0.345171 \\
\hline RAB5A & 0.343766 \\
\hline NR3C1 & 0.33937 \\
\hline TOMм5 & 0.337905 \\
\hline STK17B & 0.337529 \\
\hline PPCS & 0.337107 \\
\hline HIPK1 & 0.335792 \\
\hline RAP1A & 0.335699 \\
\hline COQ10B & 0.334904 \\
\hline UBE2B & 0.330062 \\
\hline COPS9 & 0.327904 \\
\hline MCRIP2 & 0.32762 \\
\hline UTRN & 0.327469 \\
\hline TMEM134 & 0.327162 \\
\hline PRPSAP1 & 0.325956 \\
\hline RPL36AL & 0.323148 \\
\hline DDT & 0.322227 \\
\hline NAA50 & 0.321778 \\
\hline ZC3H12A & 0.321262 \\
\hline
\end{tabular}




\begin{tabular}{|c|c|c|}
\hline PSMD9 & 0.321004 & \\
\hline IDH2 & 0.320882 & \\
\hline SLC39A8 & 0.318387 & \\
\hline PTPN7 & 0.317125 & \\
\hline PRPF4B & 0.316998 & \\
\hline TMEM256 & 0.316744 & \\
\hline FGFR1OP2 & 0.314529 & \\
\hline CYLD & 0.310099 & \\
\hline RILPL2 & 0.308712 & \\
\hline RAB11FIP1 & 0.30852 & \\
\hline SP100 & 0.308195 & \\
\hline EIF2A & 0.307811 & \\
\hline DBNL & 0.304842 & \\
\hline ELF1 & 0.302678 & \\
\hline ARPC5L & 0.301554 & \\
\hline MBP & 0.301238 & \\
\hline IDH3B & 0.30042 & \\
\hline JAK1 & 0.298506 & \\
\hline MTCH1 & 0.298415 & \\
\hline CSRNP1 & 0.298211 & \\
\hline SNX1 & 0.297778 & \\
\hline NARS & 0.297377 & \\
\hline RPL30 & 0.29459 & \\
\hline STXBP3 & 0.293635 & \\
\hline RPA3 & 0.293081 & \\
\hline PPTC7 & 0.292554 & \\
\hline ICAM3 & 0.292407 & \\
\hline ARF6 & 0.291666 & \\
\hline PCNP & 0.278957 & \\
\hline CYTH1 & 0.277775 & \\
\hline TMF1 & 0.277592 & \\
\hline SDHB & 0.275804 & \\
\hline GLUD1 & 0.274941 & \\
\hline RICTOR & 0.272747 & \\
\hline TGIF1 & 0.272536 & \\
\hline KRTCAP2 & 0.272294 & \\
\hline SUN2 & 0.271449 & \\
\hline HBP1 & 0.271284 & \\
\hline FBXW2 & 0.270081 & \\
\hline TMEM243 & 0.270012 & \\
\hline FOXP1 & 0.265435 & \\
\hline STAG2 & 0.264711 & \\
\hline SEPT1 & 0.264119 & \\
\hline GGCT & 0.263442 & \\
\hline USP16 & 0.262964 & \\
\hline PRR13 & 0.259105 & \\
\hline DENR & 0.258776 & \\
\hline YES1 & 0.258458 & \\
\hline TM9SF3 & 0.257826 & \\
\hline ТОВ2 & 0.254391 & \\
\hline CD2AP & 0.25416 & \\
\hline ALOX5AP & 0.25245 & \\
\hline CRYM & & 0.716624909 \\
\hline FRZB & & 0.687743945 \\
\hline MIA & & 0.615971774 \\
\hline GNAL & & 0.508115225 \\
\hline
\end{tabular}




\begin{tabular}{|c|c|c|c|c|c|c|c|c|c|c|c|c|}
\hline & $\begin{array}{l}\text { downregulated } \\
\text { upregulated }\end{array}$ & & & & & & & & & & & \\
\hline Gene name & Endo & T cells & Fibro A & Fibro B & SMC & NK cells & Kera A & Myeloid & Mast cells & LEC & Kera B & Melanocytes \\
\hline $\mathrm{HBB}$ & & -1.28494 & -1.39067 & -0.2907 & & -0.61282 & -0.56192 & -0.83243 & -0.63723 & 0.747558 & 1.168621 & \\
\hline CRIP1 & & & -0.54996 & & 0.420117 & 0.277647 & -0.46562 & & -0.69306 & -1.42702 & 0.667339 & \\
\hline HSPA1A & & -0.87964 & -0.49674 & -0.41027 & -0.63141 & -1.04821 & & -0.87863 & & & 0.295021 & \\
\hline RPS26 & -0.386175133 & -0.53152 & 0.257108 & & & -0.64288 & -0.64307 & -0.54053 & & -0.50426 & & \\
\hline RHOB & 0.355652103 & & -0.56797 & -0.65146 & -0.32004 & & -0.25513 & -0.60074 & & & & \\
\hline SLC38A2 & & & -0.52039 & -0.38702 & -0.33484 & & 0.260405 & -0.26639 & & & 0.383131 & \\
\hline HSPA1B & & -0.51482 & & -0.33587 & -0.47612 & -0.61661 & & -0.56837 & & & 0.373669 & \\
\hline ZFP36L2 & & -0.29358 & -0.69967 & & & -0.25094 & & -0.431 & -0.9819 & & 0.414117 & \\
\hline CD9 & & & -0.82389 & & & & -0.29616 & -0.26421 & & -0.42745 & 0.792262 & \\
\hline $\mathrm{CCL} 2$ & & & 1.17144 & 1.7037 & -0.56007 & & 1.094228 & 0.444704 & & & & \\
\hline COL1A1 & & & -0.54629 & -0.88504 & 0.365683 & & -0.75185 & & & & & -0.986448202 \\
\hline COL1A2 & & & -0.47728 & -0.73993 & 0.435566 & & -0.57827 & & & & & -0.958361919 \\
\hline CDKN1A & & & 0.281559 & 0.308844 & -0.38326 & & & & -0.31856 & & 0.439477 & \\
\hline LGALS3 & & -0.27377 & & & & & -0.36308 & -0.25732 & -0.43604 & & 1.022059 & \\
\hline MGP & 0.948557549 & & -1.08372 & -0.52398 & & & -0.4477 & & & -1.85774 & & \\
\hline IFI27 & -0.680145371 & & -0.66355 & -0.33996 & -1.0081 & & & & & & & 0.997085485 \\
\hline CD74 & & 0.303526 & -0.34686 & & & 0.468262 & & & & 0.336557 & 0.531791 & \\
\hline S100A4 & & & -0.42485 & & 0.277805 & & -0.30746 & & & & 0.551791 & -0.984496335 \\
\hline SERF2 & & & -0.34481 & -0.41265 & & & -0.26999 & & & -0.3019 & 0.593808 & \\
\hline UBC & & & -0.25354 & -0.27135 & & & & -0.33506 & -0.43605 & & 0.641359 & \\
\hline VIM & & -0.5036 & & 0.259662 & & -0.42242 & & & -0.73836 & -0.39564 & & \\
\hline MT-ND1 & 0.380233022 & & & & 0.524949 & & 0.43646 & 0.282616 & & & -0.3993 & \\
\hline S100A6 & & & -0.39955 & & & -0.29038 & -0.55178 & -0.48704 & & & 1.230911 & \\
\hline EGR1 & & & 0.410435 & 0.439865 & & & -0.25813 & & 0.338671 & & & \\
\hline RACK1 & & & 0.421725 & 0.322382 & & & -0.3689 & & & & -0.3089 & \\
\hline ATP1A1 & & & -0.45563 & -0.30995 & & & 0.331089 & & & & 0.635282 & \\
\hline TGFBI & & & 0.280441 & 0.487724 & 0.75534 & & -0.36505 & & & & & \\
\hline АСТВ & & & & & 0.425156 & 0.387227 & & 0.410863 & & & -0.68268 & \\
\hline NEAT1 & 0.30616317 & & & & 0.301384 & & 0.428777 & & -0.35999 & & & \\
\hline FOSB & & & & 0.470535 & -0.38863 & & & & 0.286919 & & 0.523372 & \\
\hline B2M & & & -0.27126 & & & & & 0.26802 & 0.42801 & & 0.818047 & \\
\hline CYR61 & 0.416500745 & & 0.283486 & 0.458588 & -0.34255 & & & & & & & \\
\hline FN1 & 0.880840823 & & -0.67044 & -0.34619 & 0.652952 & & & & & & & \\
\hline IGFBP5 & & & -0.48423 & -0.49594 & 0.433817 & & & & & 1.174626 & & \\
\hline TPM4 & & 0.260728 & -0.28955 & & 0.370445 & & & 0.315241 & & & & \\
\hline CD81 & & & & -0.31602 & & 0.279959 & -0.36293 & 0.351604 & & & & \\
\hline RPS21 & & -0.25047 & & & & -0.32775 & & & & -0.29861 & 0.270481 & \\
\hline RPL41 & & & & & & -0.30112 & & -0.29991 & & -0.27026 & 0.416271 & \\
\hline TNFSF10 & & 0.275424 & -0.26384 & & & & & & 0.407534 & & 0.401981 & \\
\hline MT-ATP8 & & & -0.49943 & -0.5045 & & & 0.347836 & & & & & -0.618700628 \\
\hline HLA-B & & & -0.40424 & & & & & 0.285737 & & 0.379249 & 0.615374 & \\
\hline DSTN & & & -0.28878 & & & -0.48812 & & & & -0.53746 & 0.486409 & \\
\hline RGCC & & 0.254525 & -0.54059 & -0.4552 & & & & & & & 0.639381 & \\
\hline FCGRT & -0.3661142 & & 0.274649 & & & & & 0.431419 & & & & \\
\hline HLA-DRB1 & -0.268887258 & & -0.39421 & & & 0.564876 & & & & & & \\
\hline RPL10 & & & 0.450716 & & & & -0.2637 & & 0.28862 & & & \\
\hline TSPO & & & & -0.30842 & & & -0.49793 & & & & 0.883512 & \\
\hline RGS1 & & 0.253246 & & & & 0.307516 & & -0.29023 & & & & \\
\hline ACTG1 & & & & 0.328335 & & & -0.5561 & & & & -1.01838 & \\
\hline CXCL14 & & & 0.475226 & 0.309929 & & & -0.29988 & & & & & \\
\hline LMNA & & & & & & & & -0.36642 & -0.56452 & & 0.343693 & \\
\hline ZFP36 & & & & & -0.25495 & & 0.350695 & & & & 1.015175 & \\
\hline IER5 & & & & & & & 0.280215 & -0.38407 & & & 0.53026 & \\
\hline socs3 & & & 0.26774 & 0.432238 & -0.29295 & & & & & & & \\
\hline AL078639.1 & & & & -0.49302 & & & 0.301421 & & & & & -0.700200821 \\
\hline ADIRF & & & -0.55759 & & & & & & & -0.43361 & 1.240828 & \\
\hline APOE & & & & 0.921339 & -0.69079 & & -0.26013 & & & & & \\
\hline FABP4 & -0.899479007 & & 0.553558 & & -0.54803 & & & & & & & \\
\hline DST & & & -0.48966 & -0.2586 & 0.251433 & & & & & & & \\
\hline PPDPF & & -0.25631 & & & & & & -0.28281 & & & 0.497682 & \\
\hline SLC25A6 & & & 0.554672 & 0.298902 & & & -0.31888 & & & & & \\
\hline FHL1 & & & -0.43854 & 0.284095 & 0.270989 & & & & & & & \\
\hline ACADVL & & & -0.2887 & -0.27407 & & & & & & & 0.452645 & \\
\hline COL3A1 & & & -0.62791 & -0.77684 & 0.436466 & & & & & & & \\
\hline FOSL2 & & 0.358867 & & & -0.25722 & & & & & & 0.420112 & \\
\hline FTH1 & & & 0.263102 & & & & & & -0.3799 & & -0.77644 & \\
\hline
\end{tabular}




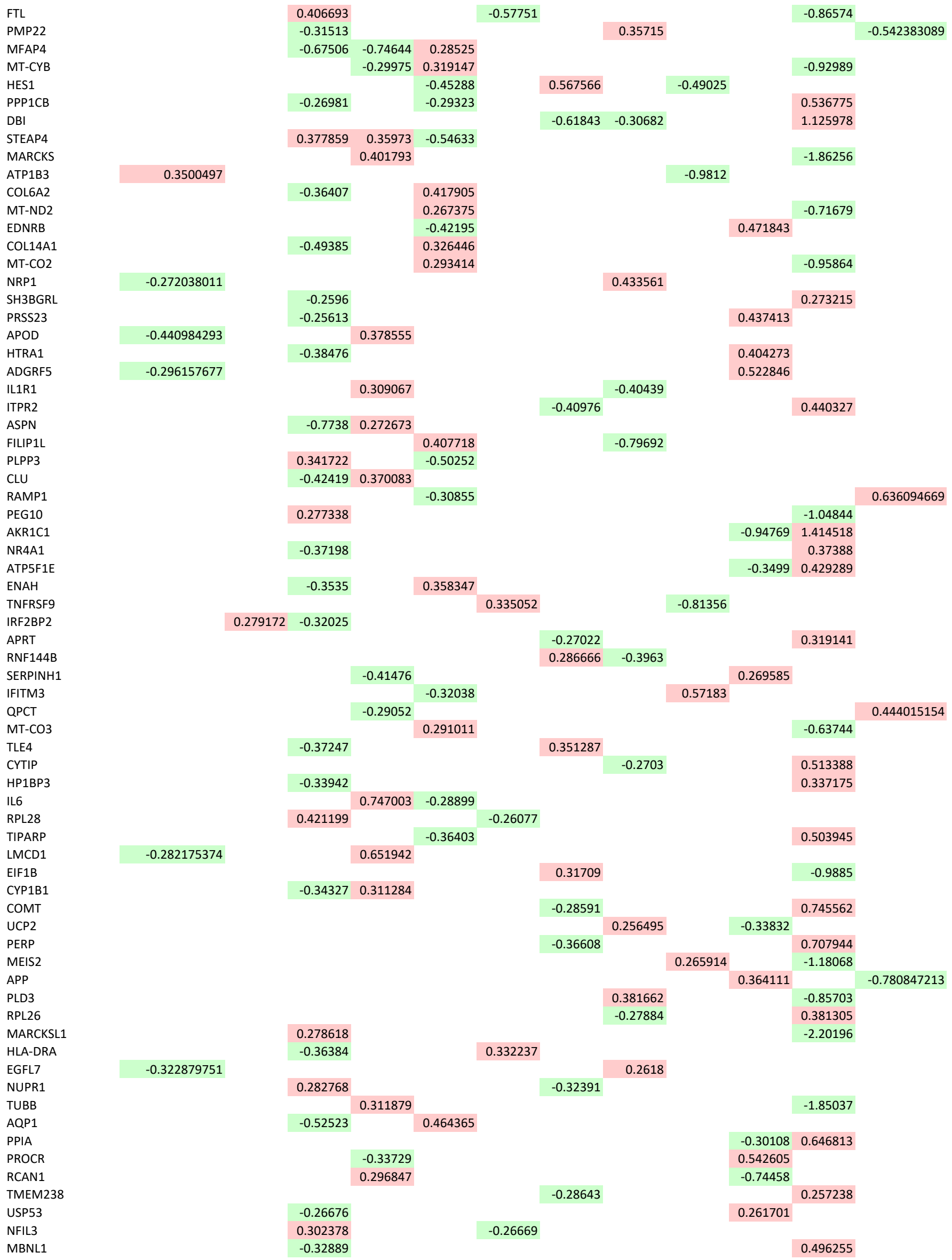


Supplementary Table 4

SDC4

JMJD1C

LDHB

GPBP1

CTSD

CIRBP

C1orf21

BTG1

LIFR

FAM129A

PDLIM3

TCF4

BASP1

SPRY1

CTGF

NCOA7

\begin{tabular}{|c|c|c|c|c|}
\hline & & \multicolumn{3}{|l|}{-0.32787} \\
\hline & & \multicolumn{3}{|l|}{0.258312} \\
\hline & & & & -0.31579 \\
\hline \multicolumn{5}{|l|}{-0.290134604} \\
\hline & -0.3215 & & 0.257248 & \\
\hline & & -0.28122 & & 0.328647 \\
\hline & & -0.37059 & & \\
\hline & & & 0.568993 & \\
\hline & & & & -0.41292 \\
\hline 0.273627518 & & -0.73301 & & \\
\hline
\end{tabular}

\begin{tabular}{|c|c|c|c|c|}
\hline-0.46125 & & & & 0.663007 \\
\hline \multicolumn{5}{|l|}{0.396796} \\
\hline & & \multicolumn{2}{|l|}{0.330715} & -1.04008 \\
\hline 0.288949 & -0.26322 & & & \\
\hline \multirow[t]{3}{*}{-0.33666} & 0.260363 & & & \\
\hline & & & & -0.56413 \\
\hline & & & -0.44271 & 0.392182 \\
\hline \multicolumn{5}{|l|}{0.438116} \\
\hline & & & 0.418976 & \\
\hline \multicolumn{5}{|l|}{0.323596} \\
\hline & & & & -2.82542 \\
\hline & & & 0.415793 & \\
\hline-0.35777 & & & & 0.609019 \\
\hline
\end{tabular}


Normalized

Name

Size Enrichment score

enrichment score NOM p-val

\section{upregulated pathways}

KEGG RIBOSOME

KEGG_FOCAL_ADHESION

KEGG_P53_SIGNALING_PATHWAY

1.9677137

0.00084848

0.001

$\begin{array}{lllll}1.7097642 & 0.00392157 & 0.03769462 & 0.073\end{array}$

$\begin{array}{llllll}0.7140249 & 1.6508062 & 0.00340716 & 0.05656531 & 0.155\end{array}$

$\begin{array}{llll}1.471174 & 0.04844961 & 0.28148794\end{array}$

$\begin{array}{rllll}1.471174 & 0.04844961 & 0.28148794 & 0.698 \\ 1.3873509 & 0.04448743 & 0.43211228 & 0.894\end{array}$

KEGG_REGULATION_OF_ACTIN_CYTOSKELETON

KEGG_CELL_CYCLE

0.45575425

0.5599457

0.5756831

0.501508

0.5075006

KEGG SMALL CELL LUNG CANCER

$\begin{array}{llll}1.3585066 & 0.09689214 & 0.43231717\end{array}$

0.894

$\begin{array}{llll}1.3516417 & 0.09756097 & 0.38852018 & 0.943\end{array}$

$\begin{array}{llll}1.3447993 & 0.0967118 & 0.35399508 & 0.946\end{array}$

0.946
0.963

KEGG_CYTOKINE_CYTOKINE_RECEPTOR_INTERACTION

0.46370274

$\begin{array}{lll}1.3247643 & 0.12222222 & 0.35443795\end{array}$

0.963

KEGG_OXIDATIVE PHOSPHORYLATION

$\begin{array}{llll}1.2875072 & 0.11568628 & 0.38972437\end{array}$

0.98

0.44854143

$\begin{array}{llll}1.2582797 & 0.1271028 & 0.41741276\end{array}$

0.993

0.5058813

KEGG_VASCULAR_SMOOTH_MUSCLE_CONTRACTION

0.45507702

$\begin{array}{lll}1.1815656 & 0.23498233 & 0.55490685\end{array}$

0.998

0.33706322

$\begin{array}{lll}1.1469922 & 0.27322406 & 0.5957508\end{array}$

0.998

0.45801345

KEGG_NOD_LIKE_RECEPTOR_SIGNALING_PATHWAY

$\begin{array}{llll}1.0708612 & 0.3222004 & 0.7352836\end{array}$

0.41684487

$\begin{array}{lll}1.061434 & 0.39784947 & 0.7113767\end{array}$

KEGG_GAP_JUNCTION

KEGG_UBIQUITIN MEDIATED_PROTEOLYSIS

0.3728257

$\begin{array}{lll}0.9917708 & 0.4877193 & 0.8355502\end{array}$

KEGG_ALZHEIMERS DISEASE

0.31610516

$\begin{array}{llll}0.8996192 & 0.62030077 & 0.96248126\end{array}$

KEGG_TIGHT_JUNCTION

KEGG_OOCYTE_MEIOSIS

KEGG GNRH SIGNALING PATHWAY

0.33862814

$\begin{array}{rrr}0.8996192 & 0.62030077 & 0.96248126 \\ 0.8609135 & 0.6666667 & 1\end{array}$

0.34411

$\begin{array}{lll}0.8483709 & 0.6841155 & 0.9790818\end{array}$

0.33358154

0.273052

0.2696637

0.3066583

KEGG_PROSTATE_CANCER

KEGG_MELANOGENESIS

$\begin{array}{lll}0.83266747 & 0.71031743 & 0.96224546\end{array}$

$\begin{array}{ll}0.7812062 & 0.8270677\end{array}$

$0.71748567 \quad 0.9067641$

$0.7097814 \quad 0.8651079$

$0.7051038 \quad 0.88014984$

0.28018788

0.25587577

KEGG_ENDOCYTOSIS

KEGG_AXON_GUIDANCE

$\begin{array}{llll}0.6754832 & 0.9158317 & 0.98398334\end{array}$

0.24902989

$\begin{array}{lll}0.64084864 & 0.93142855 & 0.9754338\end{array}$

0.1813503

$\begin{array}{llll}0.5468199 & 0.99389005 & 0.98354477\end{array}$

416 tags $=71 \%$, list $=23 \%$, signal $=89 \%$ 274 tags $=42 \%$, list $=15 \%$, signal $=48 \%$ 376 tags $=69 \%$, list $=21 \%$, signal $=86 \%$ 307 tags $=39 \%$, list $=17 \%$, signal $=46 \%$ 151 tags $=24 \%$, list $=8 \%$, signal $=25 \%$ 421 tags $=56 \%$, list $=23 \%$, signal $=72 \%$ 123 tags $=38 \%$, list $=7 \%$, signal $=40 \%$ 168 tags $=26 \%$, list $=9 \%$, signal $=28 \%$ 46 tags $=24 \%$, list $=3 \%$, signal $=24 \%$ 360 tags $=42 \%$, list $=20 \%$, signal $=52 \%$ 360 tags $=41 \%$, list $=20 \%$, signal $=51 \%$ 177 tags $=38 \%$, list $=10 \%$, signal $=41 \%$ 803 tags $=70 \%$, list $=45 \%$, signal $=124 \%$ 177 tags $=19 \%$, list $=10 \%$, signal $=21 \%$ 168 tags $=20 \%$, list $=9 \%$, signal $=22 \%$ 803 tags $=75 \%$, list $=45 \%$, signal $=134 \%$ 1089 tags $=79 \%$, list $=61 \%$, signal $=198 \%$ 694 tags $=58 \%$, list $=39 \%$, signal $=93 \%$ 151 tags $=22 \%$, list $=8 \%$, signal $=23 \%$ 745 tags $=50 \%$, list $=41 \%$, signal $=85 \%$ 803 tags $=60 \%$, list $=45 \%$, signal $=107 \%$ 316 tags $=25 \%$, list $=18 \%$, signal $=30 \%$ 180 tags $=19 \%$, list $=10 \%$, signal $=21 \%$ 803 tags $=67 \%$, list $=45 \%$, signal $=120 \%$ 694 tags $=43 \%$, list $=39 \%$, signal $=69 \%$ 173 tags $=8 \%$, list $=10 \%$, signal $=9 \%$ 697 tags $=52 \%$, list $=39 \%$, signal $=84 \%$ 890 tags $=63 \%$, list $=50 \%$, signal $=121 \%$

\section{downregulated pathways}

KEGG_CELL_ADHESION_MOLECULES CAMS

KEGG ERBB SIGNALING PATHWAY

KEGG_PHOSPHATIDYLINOSITOL_SIGNALING_SYSTEM

KEGG_FC_GAMMA_R_MEDIATED_PHAGOCYTOSIS

KEGG PROGESTERONE MEDIATED OOCYTE MATURATION

KEGG_COLORECTAL_CANCER

KEGG_GLIOMA

KEGG_LEISHMANIA INFECTION

KEGG RIG I LIKE RECEPTOR SIGNALING PATHWAY

KEGG_B_CELL_RECEPTOR_SIGNALING_PATHWAY

KEGG_VEGF_SIGNALING_PATHWAY

KEGG_JAK_STAT_SIGNALING_PATHWAY

KEGG_NATURAL_KILLER_CELL_MEDIATED_CYTOTOXICITY

KEGG_PANCREATIC_CANCER

$-0.7015002$

$\begin{array}{lll}-1.770437 & 0.00425532 & 0.10417096\end{array}$

$-0.53866065$

$-0.5603572$

$-0.517017$

$-0.51773995$

$-0.5164038$

$-0.5069$

$-0.5070932$

$-0.4998152$

$-0.4672158$

$-0.43429628$

$-0.421048$

$-0.4146357$

$\begin{array}{ll}-1.3444902 & 0.10961969\end{array}$

$-1.28640690 .17351598$

$\begin{array}{ll}-1.2772685 & 0.15384616\end{array}$

$\begin{array}{ll}-1.2240347 & 0.21412803\end{array}$

$\begin{array}{llll}-1.2201313 & 0.21786493 & 0.8893797\end{array}$

$\begin{array}{llll}-1.2012521 & 0.2516129 & 0.82415724\end{array}$

$\begin{array}{lll}-1.1863818 & 0.2553648 & 0.7653934\end{array}$

$\begin{array}{llll}-1.1541837 & 0.29777777 & 0.7748306\end{array}$

$\begin{array}{llll}-1.1305264 & 0.27959183 & 0.7630178\end{array}$

$\begin{array}{llll}-1.0375208 & 0.4245283 & 0.94654775\end{array}$

$\begin{array}{llll}-1.0289407 & 0.411111112 & 0.8919844\end{array}$

$\begin{array}{llll}-1.0049093 & 0.43949044 & 0.8877711\end{array}$

$\begin{array}{lll}-0.98968846 & 0.45073375 & 0.8653373\end{array}$

0.095

0.959

0.98

0.986

0.997

0.998

0.998

0.999

186 tags $=52 \%$, list $=10 \%$, signal $=58 \%$

113 tags $=16 \%$, list $=6 \%$, signal $=17 \%$

159 tags $=19 \%$, list $=9 \%$, signal $=20 \%$

56 tags $=15 \%$, list $=3 \%$, signal $=15 \%$

72 tags $=20 \%$, list $=10 \%$, signal $=22 \%$

113 tags $=19 \%$, list $=6 \%$, signal $=20 \%$

159 tags $=18 \%$, list $=9 \%$, signal $=19 \%$

101 tags $=24 \%$, list $=6 \%$, signal $=25 \%$

165 tags $=33 \%$, list $=9 \%$, signal $=36 \%$

56 tags $=11 \%$, list $=3 \%$, signal $=11 \%$

56 tags $=12 \%$, list $=3 \%$, signal $=12 \%$

13 tags $=17 \%$, list $=6 \%$, signal $=18 \%$

56 tags $=11 \%$, list $=3 \%$, signal $=11 \%$

56 tags $=11 \%$, list $=3 \%$, signal $=11 \%$ 
KEGG_CALCIUM_SIGNALING_PATHWAY

KEGG_LEUKOCYTE_TRANSENDOTHELIAL_MIGRATION

KEGG_EPITHELIAL_CELL_SIGNALING_IN_HELICOBACTER_PYLOR

IINFECTION

KEGG_APOPTOSIS

KEGG_CHRONIC_MYELOID_LEUKEMIA

KEGG_NEUROTROPHIN_SIGNALING_PATHWAY

KEGG SPLICEOSOME

KEGG ACUTE MYELOID LEUKEMIA

KEGG_INSULIN_SIGNALING_PATHWAY

KEGG_T_CELL_RECEPTOR_SIGNALING_PATHWAY

KEGG LONG TERM POTENTIATION

KEGG_TOLL_LIKE_RECEPTOR_SIGNALING_PATHWAY

$\begin{array}{lr}23 & -0.37924415 \\ 34 & -0.32698435 \\ & \\ 18 & -0.38236952 \\ 17 & -0.36829674 \\ 24 & -0.34108493 \\ 32 & -0.3156462 \\ 22 & -0.31649184 \\ 19 & -0.29380262 \\ 21 & -0.2697769 \\ 22 & -0.23402295 \\ 18 & -0.24559428 \\ 16 & -0.24460518\end{array}$

Significant upregulated KEGG pathways (nomalized $p$ value $<0.05$ )

Significant downregulated KEGG pathways (nomalized $p$ value $<0.05$ ) $\begin{array}{lll}-0.9752521 & 0.4896694 & 0.8431899\end{array}$

$\begin{array}{lll}-0.92310655 & 0.56646216 & 0.92095846\end{array}$

$\begin{array}{lll}-0.90741366 & 0.58099353 & 0.90281904\end{array}$

$\begin{array}{lll}-0.88370854 & 0.6441441 & 0.90603715\end{array}$

$\begin{array}{lll}-0.8645039 & 0.64551425 & 0.9025642\end{array}$

$\begin{array}{lll}-0.84622246 & 0.7070707 & 0.89410394\end{array}$

$\begin{array}{llll}-0.79836035 & 0.73473686 & 0.9409653\end{array}$

$\begin{array}{ll}-0.716207 & 0.85350317\end{array}$

$-0.6754293 \quad 0.89439654$

$\begin{array}{ll}-0.6017927 & 0.9519651\end{array}$

$\begin{array}{ll}-0.5908802 & 0.9483146\end{array}$

$\begin{array}{llll}0.5750698 & 0.93736017 & 0.96953934\end{array}$
159 tags $=13 \%$, list $=9 \%$, signal $=14 \%$

185 tags $=24 \%$, list $=10 \%$, signal $=26 \%$

188 tags $=22 \%$, list $=10 \%$, signal $=25 \%$ 179 tags $=18 \%$, list $=10 \%$, signal $=19 \%$ 113 tags $=13 \%$, list $=6 \%$, signal $=13 \%$ 179 tags $=13 \%$, list $=10 \%$, signal $=14 \%$ 155 tags $=9 \%$, list $=9 \%$, signal $=10 \%$

113 tags $=11 \%$, list $=6 \%$, signal $=11 \%$ 159 tags $=10 \%$, list $=9 \%$, signal $=10 \%$ 56 tags $=5 \%$, list $=3 \%$, signal $=5 \%$

159 tags $=11 \%$, list $=9 \%$, signal $=12 \%$

165 tags $=13 \%$, list $=9 \%$, signal $=14 \%$ 
Supplementary Table 6: VEGF signaling interaction analysis between different cell types in diabetic and non-diabetic skin cells using cell chat

\begin{tabular}{|c|c|c|c|c|c|c|c|c|c|}
\hline non-diabetic & VEGF.outdeg & VEGF.indeg & VEGF.hub & VEGF.authority & VEGF.eigen & VEGF.page_rank & VEGF.betweenness & & EGF.flowbet \\
\hline Endothelial cells & 0 & 0.168234915 & 0 & 1 & 1 & 0.389354847 & & 0 & 0 \\
\hline Fibroblasts A & 0.058494301 & 0 & 1 & & 0.618232261 & 0.081632653 & & 0 & 0 \\
\hline Fibroblasts B & 0.044476288 & 0 & 0.804189412 & & 0.504121336 & 0.081632653 & & 0 & 0 \\
\hline Keratinocytes A & 0.016411162 & 0 & 0.278044774 & 0 & 0.201841137 & 0.081632653 & & 0 & 0 \\
\hline Keratinocytes B & 0 & 0 & 0 & & 0.586620251 & 0.081632653 & & 0 & 0 \\
\hline LEC & 0 & 0.021077115 & 0 & 0.122565594 & 0.213568922 & 0.120849235 & & 0 & 0 \\
\hline Myeloid cells & 0.047925077 & 0 & 0.774837597 & & 0.489520454 & 0.081632653 & & 0 & 0 \\
\hline SMC & 0.022005203 & 0 & 0.369439027 & & 0.254672336 & 0.081632653 & & 0 & 0 \\
\hline diabetic & VEGF.outdeg & VEGF.indeg & VEGF.hub & VEGF.authority & VEGF.eigen & VEGF.page_rank & VEGF.betweenness & & EGF.flowbet \\
\hline Endothelial cells & 0 & 0.127065539 & 1.7019E-16 & & 1 & 0.401768413 & & 0 & 0 \\
\hline Fibroblasts A & 0.054565294 & 0 & 0.814778594 & $1.3267 \mathrm{E}-16$ & 0.61862797 & 0.068741699 & & 0 & 0 \\
\hline Fibroblasts B & 0.066294762 & 0 & 1 & $1.3267 \mathrm{E}-16$ & 0.755801186 & 0.068741699 & & 0 & 0 \\
\hline Keratinocytes A & 0.012709093 & 0 & 0.186711702 & $1.3267 \mathrm{E}-16$ & 0.151718845 & 0.068741699 & & 0 & 0 \\
\hline Keratinocytes B & 0 & 0 & 2.12737E-17 & $6.63348 \mathrm{E}-17$ & 0.417648981 & 0.068741699 & & 0 & 0 \\
\hline LEC & 0.002388086 & 0.081867488 & 0.041573062 & 0.654201709 & 0.685090652 & 0.185781391 & & 0 & 0.011940429 \\
\hline Myeloid cells & 0.046579599 & 0 & 0.697251144 & $1.3267 \mathrm{E}-16$ & 0.53112778 & 0.068741699 & & 0 & 0 \\
\hline SMC & 0.026396193 & 0 & 0.404073179 & $1.3267 \mathrm{E}-16$ & 0.312628291 & 0.068741699 & & 0 & 0 \\
\hline
\end{tabular}

Interaction probabilities; Rows are senders and columns are receivers

\begin{tabular}{|c|c|c|c|c|c|c|c|c|c|}
\hline & Endothelial.cells. & Fibroblasts.A.VE & Fibroblasts.B.VE & Kera & I.V Kera & & & & \\
\hline Non-diabetic & VEGF & GF & $\mathrm{GF}$ & EGF & EGF & & & Myeloid.cells.VEGF & SMC.VEGF \\
\hline Endothelial cells & 0 & & 0 & 0 & 0 & 0 & 0 & & 0 \\
\hline Fibroblasts A & 0.052166528 & & 0 & 0 & 0 & 0 & 0.006327773 & & 0 \\
\hline Fibroblasts B & 0.042309955 & & 0 & 0 & 0 & 0 & 0.002166333 & & 0 \\
\hline Keratinocytes A & 0.014484079 & & 0 & 0 & 0 & 0 & 0.001927083 & & 0 \\
\hline Keratinocytes B & 0 & & 0 & 0 & 0 & 0 & 0 & & 0 \\
\hline LEC & 0 & & 0 & 0 & 0 & 0 & 0 & & 0 \\
\hline Myeloid cells & 0.040057195 & & 0 & 0 & 0 & 0 & 0.007867882 & & 0 \\
\hline SMC & 0.019217158 & & 0 & 0 & 0 & 0 & 0.002788045 & & 0 \\
\hline
\end{tabular}

Interaction probabilities; Rows are senders and columns are receivers

\begin{tabular}{|c|c|c|c|c|c|c|c|c|c|}
\hline & Endothelial.cells. & Fibroblasts.A.VE & Fibroblasts.B.VE & Keratinocytes.A.VE & E Kera & & & & \\
\hline Diabetic & VEGF & GF & GF & GF & EGF & & & Myeloid.cells.VEGF & SMC.VEGF \\
\hline Endothelial cells & 0 & 0 & & 0 & 0 & 0 & 0 & & 0 \\
\hline Fibroblasts A & 0.032119022 & 0 & & 0 & 0 & 0 & 0.022446272 & & 0 \\
\hline Fibroblasts B & 0.040697014 & 0 & & 0 & 0 & 0 & 0.025597747 & & 0 \\
\hline Keratinocytes A & 0.006972243 & 0 & & 0 & 0 & 0 & 0.00573685 & & 0 \\
\hline Keratinocytes B & 0 & 0 & & 0 & 0 & 0 & 0 & & 0 \\
\hline LEC & 0.002388086 & 0 & & 0 & 0 & 0 & 0 & & 0 \\
\hline Myeloid cells & 0.027703482 & 0 & & 0 & 0 & 0 & 0.018876118 & & 0 \\
\hline SMC & 0.017185693 & 0 & & 0 & 0 & 0 & 0.009210501 & & 0 \\
\hline
\end{tabular}

Difference in probabilities (diabetic - non-diabetic)

Endothelial.cells.I Fibroblasts.A.VEG Fibroblasts.B.VEG Keratinocytes.A.VEc Keratinocytes.B.VE LEC.VEGF

Endothelial cells

Fibroblasts A

Fibroblasts B

Keratinocytes $A$

Keratinocytes $B$

LEC

Myeloid cells

SMC

0
-0.020047506
-0.001612941
-0.007511836
0
0.002388086
-0.012353713
-0.002031465

\footnotetext{
.
}

Myeloid.cells.VEGF SMC.VEGF

0.016118499

0.023431415

0.003809766

0
0

0.011008236

0.006422456

$\begin{array}{rll}\text { Myeloid.cells.VEGF } & \text { SMC.VEGF } & \\ 0 & 0 & 0 \\ 99 & 0 & 0 \\ 15 & 0 & 0 \\ 66 & 0 & 0 \\ 0 & 0 & 0 \\ 0 & 0 & 0 \\ 36 & 0 & 0 \\ 56 & 0 & 0\end{array}$


Supplementary Table 7: Demographics of subjects included in this study Samples used for scRNA seq study

\begin{tabular}{|c|c|c|c|c|c|c|c|c|c|c|}
\hline \multicolumn{6}{|c|}{ Diabetes } & \multicolumn{5}{|c|}{ Oral } \\
\hline Sample ID & Diabetes status & type & Gender & Age & Tissue location & BMI $\left(\mathrm{kg} / \mathrm{m}^{2}\right)$ & HbA1C (\%) & medication & Insulin & Race \\
\hline SC_NS-1 & non-diabetic & N/A & $\mathrm{F}$ & 37 & Breast & 48.5 & N/A & N/A & N/A & Caucasian \\
\hline SC_NS-2 & non-diabetic & N/A & M & 69 & Lower limb & 34.2 & N/A & N/A & N/A & African American \\
\hline SC_NS-3 & diabetic & T2DM & M & 70 & Lower limb & 26.4 & 13 & Metformin & Yes & Caucasian \\
\hline SC_NS-4 & diabetic & T2DM & $M$ & 54 & Lower limb & 27.3 & 7.3 & Metformin & Yes & Caucasian \\
\hline SC_NS-5 & diabetic & T2DM & M & 65 & Lower limb & 31.9 & 10.3 & Metformin & Yes & Caucasian \\
\hline SC_NS-6 & non-diabetic & N/A & M & 46 & Pannus & 27.2 & N/A & N/A & N/A & Caucasian \\
\hline Sc_Skin3 & non-diabetic & N/A & $\mathrm{F}$ & 23 & Breast & 42.2 & N/A & N/A & N/A & African American \\
\hline Sc_Skin4 & non-diabetic & $\mathrm{N} / \mathrm{A}$ & $\mathrm{F}$ & 28 & Breast & 26.9 & N/A & N/A & N/A & Caucasian \\
\hline \multicolumn{11}{|c|}{ Additional samples used for validation study } \\
\hline Sample ID & Diabetes status & $\begin{array}{c}\text { Diabetes } \\
\text { type }\end{array}$ & Gender & age & Tissue location & BMI $\left(\mathrm{kg} / \mathrm{m}^{2}\right)$ & HbA1C (\%) & $\begin{array}{c}\text { Oral } \\
\text { medication }\end{array}$ & Insulin & Race \\
\hline NS11 & diabetic & T2DM & M & 79 & Lower limb & 20.7 & 6.8 & No & Yes & Caucasian \\
\hline NS12 & diabetic & T2DM & M & 54 & Lower limb & 28.4 & 5.2 & Yes & No & African American \\
\hline NS13 & diabetic & T2DM & M & 55 & Pannus & 95.3 & 7.9 & N/A & N/A & Caucasian \\
\hline NS10 & non-diabetic & N/A & $\mathrm{F}$ & 53 & Lower limb & 35.2 & N/A & N/A & N/A & African American \\
\hline NS7 & non-diabetic & N/A & $M$ & 56 & Pannus & 28.2 & N/A & N/A & N/A & Caucasian \\
\hline NS14 & non-diabetic & N/A & $\mathrm{F}$ & 52 & Breast & 29.5 & N/A & N/A & N/A & Caucasian \\
\hline NS30 & non-diabetic & $\mathrm{N} / \mathrm{A}$ & $\mathrm{F}$ & 55 & Breast & 32.6 & $\mathrm{~N} / \mathrm{A}$ & N/A & $\mathrm{N} / \mathrm{A}$ & African American \\
\hline NS17 & diabetic & T2DM & M & 70 & Lower limb & 37.1 & 11.9 & N/A & Yes & Caucasian \\
\hline NS32 & diabetic & T2DM & $\mathrm{F}$ & 54 & Pannus & 55.5 & 5.4 & N/A & N/A & Caucasian \\
\hline
\end{tabular}

